\title{
Toward gas exhaustion in the W51 high-mass protoclusters $\star, \star \star$
}

\author{
A. Ginsburg ${ }^{1}$, W. M. Goss² ${ }^{2}$ C. Goddi ${ }^{3,4}$, R. Galván-Madrid ${ }^{5}$, J. E. Dale ${ }^{6}$, J. Bally ${ }^{7}$, C. D. Battersby ${ }^{8}$, A. Youngblood ${ }^{9}$,
} R. Sankrit ${ }^{10}$, R. Smith ${ }^{11}$, J. Darling ${ }^{7}$, J. M. D. Kruijssen ${ }^{13,12}$, and H. B. Liu ${ }^{1}$

${ }^{1}$ European Southern Observatory, Karl-Schwarzschild-Straße 2, 85748 Garching bei München, Germany e-mail: Adam.Ginsburg@eso.org

2 National Radio Astronomy Observatory, Socorro, NM 87801, USA

3 Department of Astrophysics/IMAPP, Radboud University Nijmegen, PO Box 9010, 6500 GL Nijmegen, The Netherlands

4 ALLEGRO/Leiden Observatory, Leiden University, PO Box 9513, 2300 RA Leiden, The Netherlands

5 Instituto de Radioastronomía y Astrofísica, UNAM, A.P. 3-72, Xangari, 58089 Morelia, Mexico

${ }^{6}$ University Observatory/Excellence Cluster "Universe" Scheinerstraße 1, 81679 München, Germany

7 CASA, University of Colorado, 389-UCB, Boulder, CO 80309, USA

8 Harvard-Smithsonian Center for Astrophysics, 60 Garden Street, Cambridge, MA 02138, USA

9 LASP, University of Colorado, 600 UCB, Boulder, CO 80309, USA

10 SOFIA Science Center, NASA Ames Research Center, M/S 232-12, Moffett Field, CA 94035, USA

11 Jodrell Bank Centre for Astrophysics, School of Physics and Astronomy, University of Manchester, Oxford Road, Manchester M13 9PL, UK

12 Max-Planck Institut für Astrophysik, Karl-Schwarzschild-Straße 1, 85748 Garching, Germany

13 Astronomisches Rechen-Institut, Zentrum für Astronomie der Universität Heidelberg, Mönchhofstraße 12-14, 69120 Heidelberg, Germany

Received 15 February 2016/ Accepted 22 June 2016

\begin{abstract}
We present new JVLA observations of the high-mass cluster-forming region W51A from 2 to $16 \mathrm{GHz}$ with resolution $\theta_{\text {fwhm }} \approx$ $0.3-0.5^{\prime \prime}$. The data reveal a wealth of observational results: (1) Currently forming, very massive (proto-O) stars are traced by o- $\mathrm{H}_{2} \mathrm{CO} 2_{1,1}-2_{1,2}$ emission, suggesting that this line can be used efficiently as a massive protostar tracer; (2) there is a spatially distributed population of $\lesssim \mathrm{mJy}$ continuum sources, including hypercompact $\mathrm{H}$ II regions and candidate colliding wind binaries, in and around the W51 proto-clusters; and (3) there are two clearly detected protoclusters, W51e and W51 IRS2, that are gas-rich but may have most of their mass in stars within their inner $\lesssim 0.05 \mathrm{pc}$. The majority of the bolometric luminosity in W51 most likely comes from a third population of OB stars between these clusters. The presence of a substantial population of exposed O-stars coincident with a population of still-forming massive stars, together with a direct measurement of the low mass loss rate via ionized gas outflow from W51 IRS2, implies that feedback is ineffective at halting star formation in massive protoclusters. Instead, feedback may shut off the large-scale accretion of diffuse gas onto the W51 protoclusters, implying that they are evolving toward a state of gas exhaustion rather than gas expulsion. Recent theoretical models predict gas exhaustion to be a necessary step in the formation of gravitationally bound stellar clusters, and our results provide an observational validation of this process.
\end{abstract}

Key words. H II regions - circumstellar matter - radio continuum: ISM - radio lines: ISM - stars: massive - stars: protostars

\section{Introduction}

The highest-mass clusters forming in our Galaxy have masses $M \gtrsim$ few $\times 10^{4} M_{\odot}$ (Portegies Zwart et al. 2010). These rare regions mark the high end of the protocluster mass function. Recent surveys have provided a complete census of all protoclusters in this high-mass range (Ginsburg et al. 2012; Urquhart et al. 2013, 2014b). As the most massive and dense star-forming regions in the Galaxy, these locations provide an important local analog to the early universe, where stars more commonly formed in clusters and generally formed in denser gas (Kruijssen 2012; Madau \& Dickinson 2014).

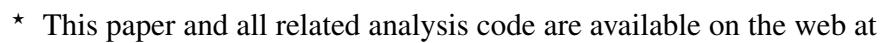
https://github.com/adamginsburg/paper_w51_evla

$\star \star$ Table A.1 and final data (FITS files) are only available at the CDS via anonymous ftp to cdsarc.u-strasbg. fr (130.79.128.5) or via http://cdsarc.u-strasbg.fr/viz-bin/qcat?]/A+A/595/A27
In such environments, feedback from massive stars is an important factor. Most star formation within high-mass clusters is likely to occur after at least one high-mass star has ignited. Many open questions remain about the effects of this feedback, as the physical complexity of such regions renders them difficult to simulate. A crucial question is whether the clusters complete the star formation process by consuming most of their gas (exhaustion) or by removing it via feedback (expulsion; Kruijssen et al. 2012; Longmore et al. 2014; Matzner \& Jumper 2015). The balance between the two processes is expected to depend on the gas volume density because higher density gas clumps are capable of evolving through a larger number of free-fall times before the gas supply is shut off by feedback, and thus higher density clumps attain larger star formation efficiencies (Kruijssen 2012). This distinction therefore governs whether the forming stars will be observable as a bound cluster or an expanding OB association. When integrated over the density probability distribution 
function of the interstellar medium in a galaxy, this argument predicts that the fraction of star formation occurring in bound clusters increases with the gas pressure and surface density, as is indeed observed (Goddard et al. 2010; Kruijssen 2012; Adamo et al. 2015).

W51 contains two of the best candidates for protoclusters that will experience gas exhaustion. This region is already extremely luminous both bolometrically and in the radio continuum. Of all the star-forming regions included in the Red MSX Survey, which is complete to sources with infrared luminosity $L>10^{4} L_{\odot}$, W51 is the most luminous (Urquhart et al. 2014a) and among the closest at a parallax-measured distance of $5.41 \mathrm{kpc}$ (Sato et al. 2010; Xu et al. 2009). The total luminosity of the W51 protocluster complex has been estimated using IRAS and KAO, $L_{\text {bol }} \sim 9.3 \times 10^{6}(D / 5.4 \mathrm{kpc})^{2} L_{\odot}$ (Harvey et al. 1986; Sievers et al. 1991). The pair of clusters in W51 providing this luminosity, W51 Main and W51 IRS2, each contain $\sim 5 \times 10^{4} M_{\odot}$ of molecular gas (Ginsburg et al. 2012).

As one of the most luminous star-forming regions in our Galaxy, W51 has been comprehensively studied in the radio and millimeter. Previous investigations have shown that the protoclusters within W51 contain many forming massive stars. Five high-mass protostars have been identified: W51 North, W51e8, W51e2w, W51e2e, W51e2nw, and W51d2 (Zhang \& Ho 1997; Keto \& Klaassen 2008; Zapata et al. 2008, 2009, 2010; Shi et al. 2010a,b; Surcis et al. 2012; Goddi et al. 2015, 2016). Another handful have already reached the main sequence and are visible in the near infrared (Goldader \& Wynn-Williams 1994; Okumura et al. 2000; Kumar et al. 2004; Barbosa et al. 2008; Figuerêdo et al. 2008).

W51 contains many distinct $\mathrm{H}$ II regions. These are classified as extended $\mathrm{H}$ II regions $(\sim 10 \mathrm{pc})$, ultracompact $\mathrm{H}$ II regions (UCH II's; $r \sim 0.1 \mathrm{pc}$ ), or hypercompact H II regions (HCH II's; $r<0.05$ pc Kurtz 2002). About ten HCH II regions, which are the youngest $\mathrm{H}$ II region class and likely contain accreting proto-OB stars (Peters et al. 2010a), have been found in W51 (Mehringer 1994). However, the census of both massive stars and protostars in W51 is incomplete.

We present observations of the inner few parsecs of the W51 protocluster forming region. We refer to the whole region, including W51 Main (which contains the W51e sources) and W51 IRS2 as "W51A". There is a shell centered on W51 Main that has a particularly bright northwest edge that is known as W51 IRS1. Other names are explained when they are used. An overview of the nomenclature used, including labels, is in Figs. 2 and A.2.

In Sect. 2, we describe the observations and data reduction. Section 3 presents the observational results, summarizing key features identified in the images and describing the point-source photometry (Sect. 3.1). Section 4 provides analysis and interpretation of the observational results. Section 5 discusses the implications of the observations. Section 6 states the conclusions.

Finally, we include the following appendices: Appendix A provides figures labeling named objects, B provides cutout images and spectral energy distribution (SED) plots for all point sources, C includes tables of observational metadata, and D shows the velocity field of the molecular gas.

\section{Observations}

We used the Karl G. Jansky Very Large Array (JVLA) in multiple bands and configurations. The observations and resulting images are summarized in Table 1. The complete observational metadata, including times of observation and program IDs, are in Table C.1. In project 12B-365, we observed in A-array in $S$ and $C$-bands ( 3 and $5 \mathrm{GHz}$, approximately) with $2 \mathrm{GHz}$ total bandwidth. In project $13 \mathrm{~A}-064$, we observed in $C$-band in $C(1 \mathrm{~h})$ and $\mathrm{A}(5 \mathrm{~h})$ arrays and in $\mathrm{Ku}-$ band in $\mathrm{D}(1 \mathrm{~h})$ and $\mathrm{B}(5 \mathrm{~h})$ arrays. We also include continuum data from project $12 \mathrm{~A}-274$ (Goddi et al. 2015, 2016) covering the range 25-36 GHz using the JVLA B array configuration.

Our spectral coverage included $\mathrm{o}-\mathrm{H}_{2} \mathrm{CO} \quad 1_{1,0}-1_{1,1}$ at $4.82966 \mathrm{GHz}$ and $2_{1,1}-2_{1,2}$ at $14.488 \mathrm{GHz}$ with $0.3 \mathrm{~km} \mathrm{~s}^{-1}$ resolution and the radio recombination lines (RRLs) $\mathrm{H} 77 \alpha$ $(14.1286 \mathrm{GHz})$ and $\mathrm{H} 110 \alpha(4.8741 \mathrm{GHz})$ at $1 \mathrm{~km} \mathrm{~s}^{-1}$ resolution. The $\mathrm{H} 110 \alpha$ line had lower $\mathrm{S} / \mathrm{N}$ than the $\mathrm{H} 77 \alpha$ line but was otherwise similar; it is not discussed further in this paper.

Data reduction was performed using $\mathrm{CASA}^{1}$ (McMullin et al. 2007). The pipeline-calibrated products were used, then imaging was performed using CLEAN. For most images discussed here, we used uniform weighting. The reduction scripts are included in a repository https://github.com/ adamginsburg/w51evlareductionscripts.

We did not achieve a thermal-noise-limited image in any of the 13A-064 data. For example, in the $C$-band A-array data, the thermal noise is $\sim 5 \mu \mathrm{Jy}$, while the achieved RMS is $\sim 30-60 \mu \mathrm{Jy}$ depending on location in the image. There is a large amount of resolved-out structure in the images that hinders cleaning. The images are generally artifact-dominated at the low-signal end, where inadequately cleaned sidelobes of bright features provide significant non-Gaussian noise. Some of these artifacts can be seen in the $C$-band image, Fig. 2 (or Fig. A.1 without annotations).

Despite the deficiency in the image quality, the new observations are $\sim 5 \times$ more sensitive than any previous observations. The new observations also cover a much broader range of frequencies than those performed previously with the VLA. Table 1 gives the estimated noise in each image and an estimate of the dynamic range, which is the peak intensity in the image divided by the noise measured in a signal-free region of the image.

Figures 1 and 2 show the best-quality images in the $\mathrm{Ku}$-band and $C$-band combining the short and long array configurations. These images were made from a combination of the full bandwidth in their respective bands. They were used in the figures in the main text of the paper and for source identification but not for photometry. Instead, for the 13A-064 data, individual $1 \mathrm{GHz}$ bands including only the longer-baseline configuration were used to measure the intensity of the identified sources.

For the spectral line analysis, we used the cubes listed in Table 2. For the $\mathrm{Ku}$-band naturally-weighted cube, the noise is higher than in the robust-weighted cube because large angular scale artifacts are prevalent, especially at the image edges.

In some figures, we show unsharp-masked versions of the data. These are equivalent to images made excluding short baselines, but we instead performed unsharp masking (subtracting a Gaussian-smoothed version of the image from itself) in the image domain for computational convenience.

\section{Observational results}

We report seven distinct observational results: (i) the detection of new continuum sources, including hypercompact $\mathrm{H}$ II regions and probable colliding wind binaries (Sect. 3.1); (ii) the detection of variability in some of the faint point sources over a $20 \mathrm{yr}$ timescale (Sect. 3.1.1); (iii) the detection of radio continuum sources associated with near-infrared sources, most

\footnotetext{
http://casa.nrao.edu
} 
Table 1. Observations.

\begin{tabular}{cccccccc}
\hline \hline Epoch & $\begin{array}{c}\text { Frequency } \\
\text { GHz }\end{array}$ & $\begin{array}{c}\text { BMAJ } \\
\prime \prime\end{array}$ & $\begin{array}{c}\text { BMIN } \\
\prime \prime\end{array}$ & $\begin{array}{c}\text { BPA } \\
\circ\end{array}$ & $\begin{array}{c}\text { Noise estimate } \\
\text { mJy beam }^{-1}\end{array}$ & Dynamic range & Jy-Kelvin \\
\hline 2 & 2.5 & 0.54 & 0.53 & -175 & 0.20 & 54 & $6.8 \times 10^{5}$ \\
2 & 3.5 & 0.41 & 0.39 & 0 & 0.06 & 190 & $6.3 \times 10^{5}$ \\
1 & 4.9 & 0.45 & 0.38 & 88 & 0.18 & 150 & $3 \times 10^{5}$ \\
2 & 4.9 & 0.32 & 0.29 & -84 & 0.04 & 320 & $5.6 \times 10^{5}$ \\
3 & 4.9 & 0.33 & 0.26 & 68 & 0.06 & 200 & $5.9 \times 10^{5}$ \\
3 & 5.5 & 0.29 & 0.22 & 80 & 0.03 & 420 & $6.4 \times 10^{5}$ \\
2 & 5.9 & 0.27 & 0.23 & -81 & 0.03 & 460 & $5.7 \times 10^{5}$ \\
3 & 5.9 & 0.31 & 0.19 & 74 & 0.03 & 420 & $6 \times 10^{5}$ \\
1 & 8.4 & 0.47 & 0.39 & 82 & 0.08 & 860 & $9.5 \times 10^{4}$ \\
2 & 12.6 & 0.38 & 0.35 & 83 & 0.08 & 1200 & $5.7 \times 10^{4}$ \\
2 & 13.4 & 0.34 & 0.33 & 14 & 0.05 & 1800 & $6 \times 10^{4}$ \\
2 & 14.1 & 0.34 & 0.33 & 68 & 0.09 & 1200 & $5.6 \times 10^{4}$ \\
1 & 22.5 & 0.32 & 0.29 & -84 & 0.57 & 280 & $2.6 \times 10^{4}$ \\
2 & 25.0 & 0.28 & 0.24 & -4 & 0.64 & 300 & $2.8 \times 10^{4}$ \\
2 & 27.0 & 0.25 & 0.22 & 56 & 1.13 & 140 & $3.1 \times 10^{4}$ \\
2 & 29.0 & 0.23 & 0.21 & 63 & 1.32 & 86 & $3 \times 10^{4}$ \\
2 & 33.0 & 0.21 & 0.18 & 54 & 1.11 & 130 & $2.9 \times 10^{4}$ \\
2 & 36.0 & 0.18 & 0.17 & 74 & 1.23 & 72 & $3.1 \times 10^{4}$ \\
\hline
\end{tabular}

Notes. Jy-Kelvin gives the conversion factor from Jy to Kelvin given the synthesized beam size and observation frequency.

Table 2. Spectral cubes.

\begin{tabular}{|c|c|c|c|c|c|c|c|c|}
\hline Cube ID & $\begin{array}{l}\text { Frequency } \\
\mathrm{GHz}\end{array}$ & $\begin{array}{l}\text { Channel width } \\
\mathrm{km} \mathrm{s}^{-1}\end{array}$ & $\begin{array}{l}\text { rms } \\
\text { mJy beam }^{-1}\end{array}$ & $\begin{array}{l}\mathrm{rms}_{K} \\
\mathrm{~K}\end{array}$ & $\begin{array}{l}\text { BMAJ } \\
\prime \prime\end{array}$ & $\begin{array}{l}\text { BMIN } \\
\prime \prime\end{array}$ & $\begin{array}{l}\text { BPA } \\
\circ\end{array}$ & Jy-Kelvin \\
\hline 1-1 Uniform & 4.829660 & 0.50 & 1.03 & 63 & 0.51 & 0.35 & 84 & $6.1 \times 10^{4}$ \\
\hline 1-1 Natural & 4.829660 & 0.50 & 0.56 & $1.6 \times 10^{2}$ & 1.06 & 0.81 & 52 & $2.9 \times 10^{5}$ \\
\hline H77 $\alpha$ Briggs 0 & 14.128610 & 1.33 & 0.33 & 12 & 0.41 & 0.42 & 13 & $3.6 \times 10^{4}$ \\
\hline 2-2 Natural & 14.488479 & 0.50 & 1.28 & 7.6 & 1.13 & 0.87 & 57 & $5.9 \times 10^{3}$ \\
\hline 2-2 Briggs 0 & 14.488479 & 0.50 & 0.50 & 17 & 0.43 & 0.42 & 20 & $3.3 \times 10^{4}$ \\
\hline
\end{tabular}

Notes. Jy-Kelvin gives the conversion factor from Jy to Kelvin given the synthesized beam size and observation frequency.

likely un-embedded O-stars (Sect. 3.1.2); (iv) the detection of $\mathrm{H}_{2} \mathrm{CO} 2_{1,1}-2_{1,2}$ emission around sources e2, e8, and W51 North (Sect. 3.2); (v) measurements of line-of-sight velocities toward many ultracompact $\mathrm{H}$ II regions using $\mathrm{H} 77 \alpha$ and/or $\mathrm{H}_{2} \mathrm{CO}$ (Sect. 3.3); (vi) detection of Orion-bar-like sharp edges to the W51 Main/W51 IRS1 H II region, most likely tracing photondominated regions (PDRs) (Sect. 3.4); and (vii) detection of a $\sim 0.1 \mathrm{pc}$ ionized outflow in $\mathrm{H} 77 \alpha$ that had previously been identified in mid-infrared ionized line emission (Sect. 3.5).

\subsection{Continuum sources and photometry}

We report new detections of several sources and concrete identifications of others that were detected in previous data sets but not reported.

We follow the naming scheme introduced by Mehringer (1994). For the compact $\left(r<1^{\prime \prime}\right)$ sources within 1 arcmin of W51e2, we use the name W51e followed by a number. We identify two new sources, e9 and e10, which were previously detected but never named. We split source e8 into a north and south component, plus a more extended molecular component e8mol. We also identify a molecular component between e1, e8, and e10: e10mol. The source positions and approximate radii for resolved continuum sources are shown in Fig. 2 and listed in
Table 3. The positions and radii are estimated by eye; they are not fits to the data.

We include in this catalog any pointlike sources (at $\sim 0.2-0.4^{\prime \prime}$ resolution) with emission in two bands, $\mathrm{Ku}$ and $C$ (14 and $5 \mathrm{GHz}$; Table 4). To identify point sources, we used uniformly-weighted images. These images exclude much of the extended emission, which makes point source detection more straightforward. There is some reason for concern about this approach, since it can make local peaks of the diffuse emission appear to be pointlike. However, for many of the faint and low-significance peaks, we have found clear near-infrared associations (Sect. 3.1.2) and have detected the sources in multiple bands, suggesting that these point sources are real.

The point source photometry data are in Table 4. The Epoch column describes the data source: Epoch 1 comes from Mehringer (1994), Epoch 2 comes from 12A-274, 12B-365, or 13A-064 (mid 2012-early 2013), and Epoch 3 (C-band only) comes from 13A-064 in 2014. For the multi-configuration combined images, we list the date of the highest-resolution observations. Full details of the data provenance are given in Table C.1.

There are two intensity columns in Table 4 . The first shows the peak intensity within a circular aperture with a radius equal to the beam major FWHM. The second column, "Peak - Background", shows the peak intensity minus the minimum 


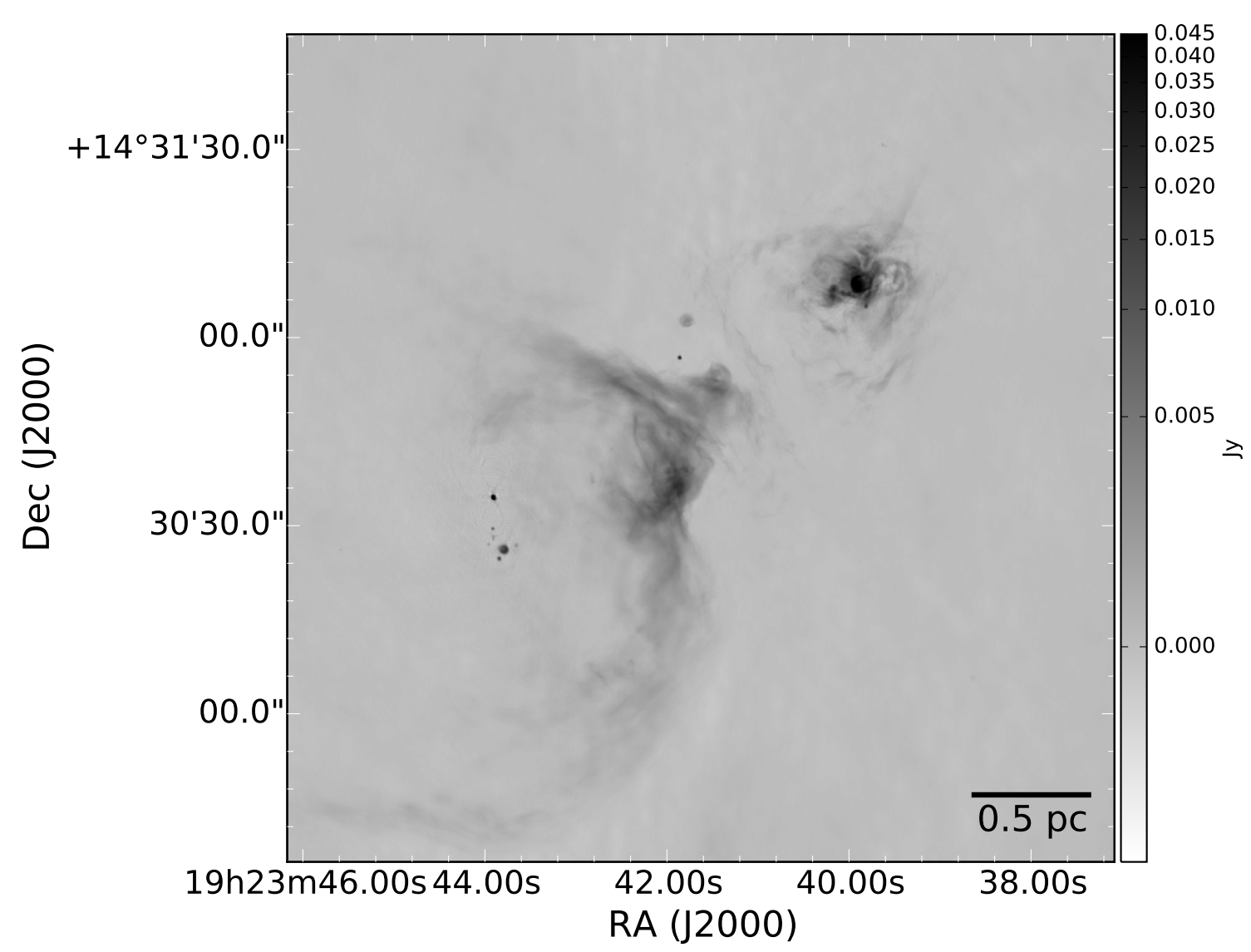

Fig. 1. Ku-band image (14.5 GHz, tracing ionized gas) of the W51 region produced with a combination of JVLA B and D array data using uniform weighting. The synthesized beam size is $\theta_{\mathrm{FWHM}} \approx 0.33^{\prime \prime}$. The compact, circular region in the upper-right corner is IRS2, while the more diffuse bright region in the center is IRS1, marking the edge of the W51 Main shell. Compact sources are identified in Fig. 2.

intensity in a box $6 \times 6$ beam FWHMs. This backgroundsubtracted intensity is meant to account for negative bowling that affects parts of the images; there are cases in which a bright point source is seen on a deeply negative background such that the measured peak intensity is near zero. When these two values agree, they are reliable, but when they differ significantly, they are probably affected by image reconstruction artifacts and the background-subtracted version is preferred.

The RMS column gives the error measured in a signal-free region of the image. It is approximately the same as that shown in Table 1, though slightly different regions are used in each case.

\subsubsection{Variability}

The multi-epoch data demonstrate that at least one of these sources is variable. The most convincing case for variability is in the (double) source d4. In the Mehringer (1994) data, there is no hint of emission at this location, with a $3 \sigma$ upper limit of $0.6 \mathrm{mJy}$. At the same position and frequency in 2014 , there is a $1.03 \pm 0.06 \mathrm{mJy}$ source at the position of $\mathrm{d} 4 \mathrm{e}$.

\subsubsection{Multi-wavelength source associations}

We compare our extracted source catalog with the MOXC Chandra X-ray catalog (Townsley et al. 2014) and the UKIDSS Galactic Plane Survey (UGPS; Lucas et al. 2008) to identify sources that are detected at multiple wavelengths. The associations are listed in Table 8. We discuss some individual source associations in more detail in this section. The absolute astrometric offsets in the Chandra catalogs are $<0.1^{\prime \prime}$ (Townsley et al. 2014), and typical absolute astrometric errors in JVLA data ${ }^{2}$ are $\sim 10 \%$ of the beam, or in our case $\lesssim 0.02-0.04^{\prime \prime}$.

Both lobes of the variable source $\mathrm{d} 4$ are $0.3^{\prime \prime}$ from MOXC $192339.62+143130.3$ and d6 is $0.3^{\prime \prime}$ from MOXC $192341.23+143111.8\left(0.3^{\prime \prime}\right.$ is about a full-width resolution element in the radio data, so this offset is highly significant). These separations may indicate that either the H II regions or the MOXC point sources are associated with diffuse material, e.g., outflows, near stars rather than the stars themselves. Candidate sources e20 and e22 are $<0.1^{\prime \prime}$ from MOXC $192342.86+143027.5$ and 192342.77+143027.5, respectively. There are a handful of X-ray sources within W51 IRS2 that could be associated with faint radio sources that we are unable to detect against the bright diffuse background emission, but these X-ray sources could also be associated with outflowing material (e.g., the Lacy jet; see Sect. 3.5). The lack of correlation between $\mathrm{X}$-ray and $\mathrm{HCH}$ II regions indicates that the embedded OB stars within these $\mathrm{HCH}$ II regions are at most weak X-ray emitters.

We compared our point source catalog to the UKIDSS $K$ band and UWISH2 $\mathrm{H}_{2}$ images to search for infrared associations with our detected radio sources (Lucas et al. 2008; Froebrich et al. 2011). Most of the W51e sources have no association, as is expected given the high extinction in this region $\left(A_{V}>100\right.$, based on mm emission). We discuss the exceptions here. Diffuse source e 7 to the south is associated with

\footnotetext{
2 https://science.nrao.edu/facilities/vla/docs/
} manuals/oss/performance/positional-accuracy 


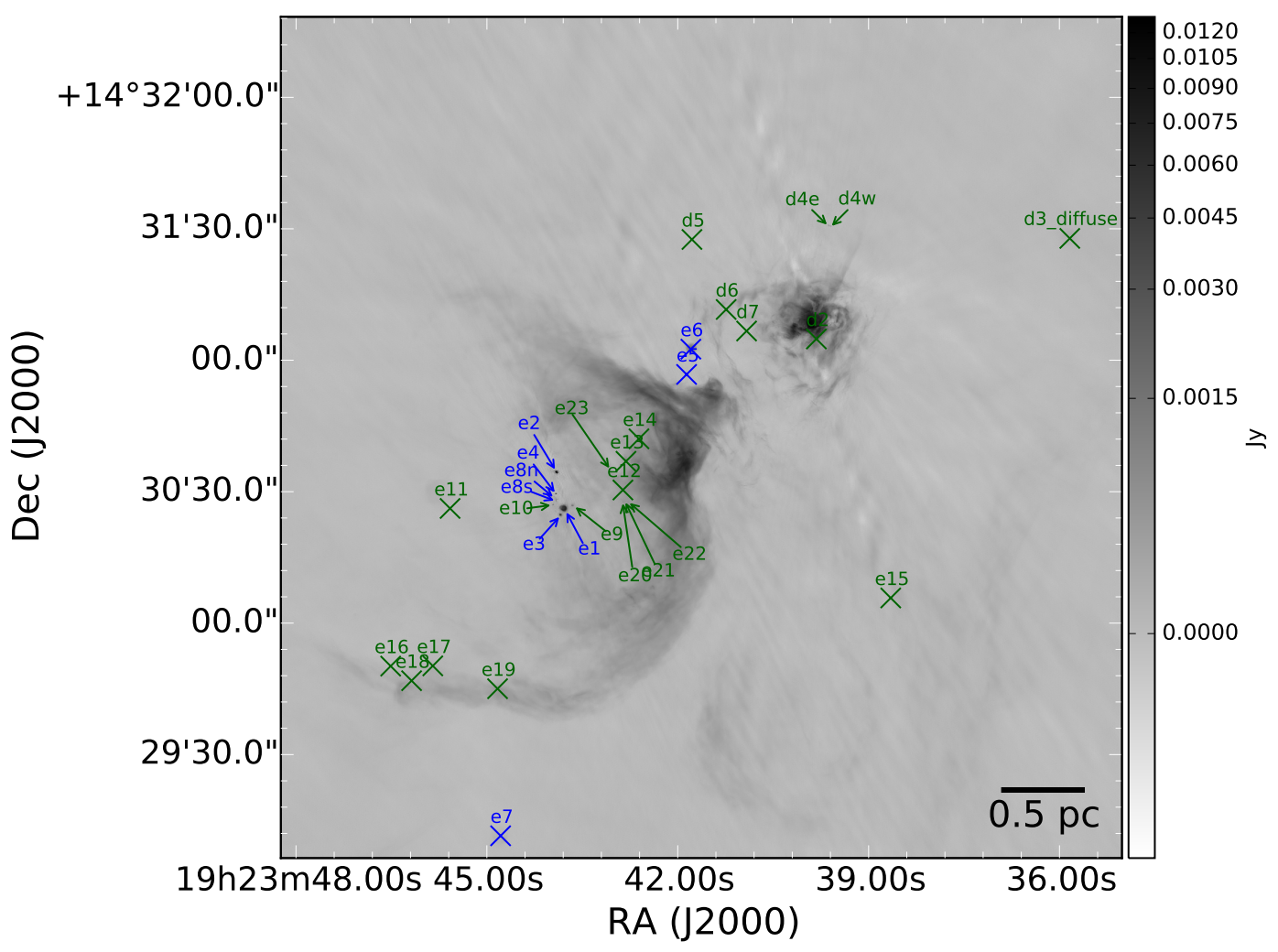

Fig. 2. $C$-band image (4.9 GHz, tracing ionized gas) of the W51A region with point sources labeled. The blue sources are previously known; the green are new additions in this paper. The synthesized beam size is $\theta_{\text {FWHM }} \approx 0.3^{\prime \prime}$.

UGPS J192344.79+142911.2 (Lucas et al. 2008). Three sources, e20, e21, and e22, have NIR $K$-band counterparts, though the UGPS cataloged positions do not align with the evident NIR $K$-band sources in the archive images. We nonetheless consider these sources to have infrared counterparts; likely adaptive optics imaging will be required to obtain reliable flux density measurements for these due to both point source and extended emission confusion in the region. Similarly, e5 exhibits some NIR $K$-band emission, though because it is diffuse, this emission is not cataloged in the UGPS.

The source $\mathrm{d} 7$ is associated with a luminous infrared source, which is seen both in UKIDSS (Lucas et al. 2008) and NACO (Figuerêdo et al. 2008) images. However, the reported position of the near-infrared $K$-band Goldader \& Wynn-Williams (1994) source RS15 is $0.7^{\prime \prime}$ away, even though in the UKIDSS images there is no detected emission at this position. Surprisingly, the associated MOXC Chandra source (Townsley et al. 2014) is more closely aligned with the offset RS15 position than the true source location in the NACO and UKIDSS images. High proper motion is unlikely to be the explanation for this discrepancy, since the source would have to be moving at $v \sim 10^{3} \mathrm{~km} \mathrm{~s}^{-1}$ to have shifted $0.7^{\prime \prime}$ in $30 \mathrm{yr}$. Despite this confusion, we regard the NIR, X-ray, and radio features to have a common origin.

\section{2. $\mathrm{H}_{2} \mathrm{CO} 2_{1,1}-2_{1,2}$ emission}

We detect $\mathrm{H}_{2} \mathrm{CO} 2_{1,1}-2_{1,2}$ emission around W51e2, W51e8, in a region between $\mathrm{e} 1, \mathrm{e} 8$, and e10, and in W51 North. We report the tentative detection of an extended structure between e 2 and $\mathrm{e} 1$, though this structure is weak and diffuse. The fitted emission line parameters are listed in Table 5. The emission is shown in Figs. 12, 14, and 15 and discussed in Sect. 4.3.

\subsection{Line-of-sight velocities}

We have detected $\mathrm{H} 77 \alpha$ emission from many of the ultracompact and hypercompact H II regions within W51. We report their lineof-sight velocities as measured from Gaussian profile fits to their extracted spectra.

The $\mathrm{H} 77 \alpha$ emission line parameters are listed in Table 7, and the $\mathrm{p}-\mathrm{H}_{2} \mathrm{CO} 2_{1,1}-2_{1,2}$ absorption line parameters are in Table 6.

The $\mathrm{H}_{2} \mathrm{CO}$ absorption lines provide velocities of foreground molecular gas. The e5 and e6 sources are close to one another (separation $3.1^{\prime \prime}$, or projected distance $0.08 \mathrm{pc}$ ), and both exhibit $\mathrm{H}_{2} \mathrm{CO}$ absorption at $62-63 \mathrm{~km} \mathrm{~s}^{-1}$. Source e6 is detected in $\mathrm{H} 77 \alpha$ at $68 \mathrm{~km} \mathrm{~s}^{-1}$, suggesting that the true velocity of both e5 and $\mathrm{e} 6$ are redshifted from the $\mathrm{H}_{2} \mathrm{CO}$ absorption lines.

\subsection{Diffuse continuum emission features}

There are a few new notable continuum emission features detected in our data that were not detected in previous shallower data.

Near source e11, there is a bow-shaped feature (Fig. 3). There are no known associated sources at other wavelengths, though in the Spitzer GLIMPSE (Benjamin et al. 2003) images, there is some diffuse emission at this location. The bow-like structure points away from e11, suggesting that e11 produces the bow-shaped nebula.

The source $\mathrm{d} 3$ is an $\mathrm{H}$ II region with radius $r \sim 1.9^{\prime \prime}$. It is associated with a Spitzer source and the 2MASS source 2MASX $\mathrm{J} 19233591+1431288$. The sources d3, e1, e6, and e7 are compact but resolved $(0.02-0.07 \mathrm{pc})$ and round; they are therefore classified as ultracompact $\mathrm{H}$ II regions. 
Table 3. Source positions.

\begin{tabular}{|c|c|c|c|c|c|c|}
\hline Source name & RA & Dec & $\begin{array}{r}\text { Radius } \\
\prime \prime\end{array}$ & $\begin{array}{r}\text { Phys. radius } \\
\text { pc }\end{array}$ & SED class & Classification \\
\hline Between & $19: 23: 41.47$ & $14: 30: 51.0$ & 4.5 & 0.11 & $E$ & - \\
\hline G49.46-0.36 & $19: 23: 35.12$ & $14: 29: 55.8$ & 34.4 & 0.85 & $E$ & - \\
\hline G49.46-0.38 & $19: 23: 40.18$ & $14: 29: 39.1$ & 19.1 & 0.47 & $E$ & - \\
\hline W51 IRS 2 & $19: 23: 39.89$ & $14: 31: 08.3$ & 1.3 & 0.03 & $E$ & - \\
\hline W51 IRS 2 bubble & $19: 23: 39.90$ & $14: 31: 08.5$ & 21.4 & 0.53 & $E$ & - \\
\hline W51 Main 40 kms RRL Shell & $19: 23: 42.23$ & $14: 30: 40.2$ & 7.8 & 0.19 & $E$ & - \\
\hline W51 Main 65 kms RRL shell & $19: 23: 42.55$ & $14: 30: 43.1$ & 10.8 & 0.27 & $E$ & - \\
\hline W51 Main Shell & $19: 23: 44.68$ & $14: 30: 23.5$ & 41.5 & 1.03 & $E$ & - \\
\hline W51G 8 & $19: 23: 50.69$ & $14: 32: 50.1$ & 7.1 & 0.18 & $E$ & - \\
\hline $\operatorname{arc}$ & $19: 23: 41.00$ & $14: 30: 37.3$ & 6.0 & 0.15 & $E$ & - \\
\hline $\mathrm{d} 2$ & $19: 23: 39.82$ & $14: 31: 04.9$ & - & - & $b, d, n$ & HCHII \\
\hline d3 & $19: 23: 35.87$ & $14: 31: 27.9$ & 1.9 & 0.05 & $E$ & UCHII \\
\hline $\mathrm{d} 4 \mathrm{e}$ & $19: 23: 39.64$ & $14: 31: 30.6$ & - & - & $f, v$ & cCWB \\
\hline $\mathrm{d} 4 \mathrm{w}$ & $19: 23: 39.60$ & $14: 31: 30.4$ & - & - & $f$ & cCWB \\
\hline d5 & $19: 23: 41.78$ & $14: 31: 27.6$ & - & - & $w$ & cCWB \\
\hline d6 & $19: 23: 41.24$ & $14: 31: 11.6$ & - & - & $f, n$ & cCWB \\
\hline $\mathrm{d} 7$ & $19: 23: 40.92$ & $14: 31: 06.7$ & - & - & $f$ & $\mathrm{cCWB}$ \\
\hline e1 & $19: 23: 43.78$ & $14: 30: 26.1$ & 0.7 & 0.02 & $E$ & UCHII \\
\hline e2 & $19: 23: 43.91$ & $14: 30: 34.6$ & - & - & $a, d$ & HCHII \\
\hline e3 & $19: 23: 43.84$ & $14: 30: 24.7$ & - & - & $b, d$ & HCHII \\
\hline e4 & $19: 23: 43.91$ & $14: 30: 29.5$ & - & - & $b, d$ & HCHII \\
\hline e5 & $19: 23: 41.86$ & $14: 30: 56.8$ & - & - & $b, d$ & HCHII \\
\hline e6 & $19: 23: 41.79$ & $14: 31: 02.7$ & 1.0 & 0.03 & $E$ & UCHII \\
\hline e7 & $19: 23: 44.79$ & $14: 29: 11.3$ & 1.8 & 0.04 & $E$ & UCHII \\
\hline$e 8 n$ & $19: 23: 43.91$ & $14: 30: 28.2$ & - & - & $b / w$ & HCHII \\
\hline e8s & $19: 23: 43.91$ & $14: 30: 27.9$ & - & - & $b$ & HCHII \\
\hline e9 & $19: 23: 43.65$ & $14: 30: 26.8$ & - & - & $c, f$ & HCHII \\
\hline $\mathrm{e} 10$ & $19: 23: 43.96$ & $14: 30: 27.0$ & - & - & $f$ & HCHII \\
\hline e11 & $19: 23: 45.58$ & $14: 30: 26.2$ & - & - & $b$ & HCHII \\
\hline e11d & $19: 23: 45.69$ & $14: 30: 29.1$ & 3.6 & 0.09 & $E$ & HII \\
\hline e12 & $19: 23: 42.86$ & $14: 30: 30.4$ & - & - & $E$ & - \\
\hline e13 & $19: 23: 42.81$ & $14: 30: 36.9$ & - & - & $b, c, E$ & $\mathrm{cCWB} / \mathrm{HII}$ \\
\hline e14 & $19: 23: 42.61$ & $14: 30: 42.1$ & - & - & $b, c, E$ & $\mathrm{cCWB} / \mathrm{HII}$ \\
\hline e15 & $19: 23: 38.65$ & $14: 30: 05.7$ & - & - & $b, c, E$ & UCHII \\
\hline e16 & $19: 23: 46.51$ & $14: 29: 50.2$ & - & - & $E$ & - \\
\hline e17 & $19: 23: 45.85$ & $14: 29: 50.2$ & - & - & $E$ & - \\
\hline e18 & $19: 23: 46.18$ & $14: 29: 46.9$ & - & - & $E$ & - \\
\hline e18d & $19: 23: 46.18$ & $14: 29: 44.0$ & 3.2 & 0.08 & $E$ & HII \\
\hline e19 & $19: 23: 44.83$ & $14: 29: 45.0$ & - & - & $E$ & - \\
\hline $\mathrm{e} 20$ & $19: 23: 42.86$ & $14: 30: 27.6$ & - & - & $E$ & - \\
\hline e21 & $19: 23: 42.83$ & $14: 30: 27.8$ & - & - & $E$ & - \\
\hline $\mathrm{e} 22$ & $19: 23: 42.78$ & $14: 30: 27.6$ & - & - & $E$ & - \\
\hline $\mathrm{e} 23$ & $19: 23: 43.06$ & $14: 30: 34.9$ & - & - & $E$ & - \\
\hline
\end{tabular}

Notes. Objects with name e\#d are the diffuse counterparts to point sources. The absolute positional accuracy is $\sim 0.2^{\prime \prime}$. Sources with no radius are unresolved, with upper limits of $0.3^{\prime \prime}(0.007 \mathrm{pc})$.Source names correspond to the labels in Figs. 2 and A.2. $a$ : $v^{2}$ dependence. $b$ : $v^{1}$ dependence. $c$ : $v<5 \mathrm{GHz}$ excess or negative index. $d: v>15 \mathrm{GHz}$ flat. $f: v$-independent flat. $E$ : extended (photometry not trustworthy). $n$ : near bright, extended emission (may affect photometry). $v$ : Likely variable. $w$ : Too weak for SED classification. HCHII: hypercompact H II region. UCHII: ultracompact $\mathrm{H}$ II region. HII: extended $\mathrm{H}$ II region or part of a larger $\mathrm{H}$ II region. cCWB: candidate colliding-wind binaries.

The diffuse emission associated with the W51 Main shell and W51 IRS1 traces a broad arc $\left(r \sim 42^{\prime \prime}\right)$ that has been observed in many previous data sets. The new deeper, higher-resolution data represent a substantial improvement in image quality. While in previous observations a relatively smooth and clumpy structure was observed, the new images reveal a network of wispy, sharpedged, filamentary structures. Figure 4 shows W51 IRS1, the region between the W51e and W51 IRS2 clusters. While this area contains few clear individual sources, it accounts for the majority of the radio luminosity of the W51 Main region and somewhere between one quarter and one half the bolometric luminosity of the whole W51A complex.

The filamentary structures in W51 IRS1 are unresolved along the short axis, with aspect ratios $>25$. These resemble the "stringlike ionized features" noted by Yusef-Zadeh $(1990)^{3}$ in Orion, which are associated primarily with the Orion Bar PDR

3 See http://images.nrao.edu/402/. 
Table 4. Continuum point sources (excerpt).

\begin{tabular}{|c|c|c|c|c|c|c|}
\hline Object & Epoch & Obs. date & $\begin{array}{c}\text { Peak } S_{v} \\
\text { mJy beam }^{-1}\end{array}$ & $\begin{array}{c}\text { Peak - background } \\
\text { mJy beam }^{-1}\end{array}$ & $\begin{array}{c}\mathrm{rms} \\
\text { mJy beam }^{-1}\end{array}$ & $\begin{array}{c}\text { Frequency } \\
\mathrm{GHz}\end{array}$ \\
\hline $\mathrm{d} 2$ & 2 & 2012-10-16 & 5.5 & 7.7 & 0.2 & 2.5 \\
\hline $\mathrm{d} 2$ & 1 & 1993-03-16 & 10 & 10 & 0.5 & 22.5 \\
\hline d3-diffuse & 1 & $1992-10-25$ & 0.3 & 0.3 & 0.2 & 4.9 \\
\hline d3-diffuse & 2 & 2012-06-21 & - & - & 1 & 33.0 \\
\hline $\mathrm{d} 4 \mathrm{e}$ & 2 & 2013-03-02 & 1 & 1.3 & 0.1 & 12.6 \\
\hline $\mathrm{d} 4 \mathrm{w}$ & 2 & $2012-10-16$ & 0.51 & 0.79 & 0.05 & 4.9 \\
\hline $\mathrm{d} 4 \mathrm{w}$ & 2 & 2012-06-21 & 1 & 4 & 1 & 27.0 \\
\hline $\mathrm{d} 5$ & 2 & $2012-10-16$ & -0.03 & 0.11 & 0.04 & 5.9 \\
\hline d6 & 2 & $2012-10-16$ & 1 & 1.4 & 0.2 & 2.5 \\
\hline d6 & 1 & 1993-03-16 & 0.6 & 1.4 & 0.5 & 22.5 \\
\hline $\mathrm{d} 7$ & 3 & 2014-04-19 & 0.24 & 0.38 & 0.08 & 4.9 \\
\hline d7 & 2 & $2012-06-21$ & 1 & 1 & 1 & 33.0 \\
\hline $\mathrm{e} 1$ & 2 & 2013-03-02 & 23 & 23 & 0.1 & 12.6 \\
\hline e10 & 2 & $2012-10-16$ & 1.7 & 2 & 0.05 & 4.9 \\
\hline e10 & 2 & 2012-06-21 & 2 & 5 & 1 & 27.0 \\
\hline e11 & 2 & $2012-10-16$ & 0.29 & 0.38 & 0.04 & 5.9 \\
\hline e12 & 2 & $2012-10-16$ & -0 & 0.9 & 0.2 & 2.5 \\
\hline $\mathrm{e} 12$ & 1 & 1993-03-16 & 0.2 & 1.2 & 0.5 & 22.5 \\
\hline e13 & 2 & $2012-10-16$ & 0.76 & 0.97 & 0.05 & 4.9 \\
\hline e13 & 2 & 2012-06-21 & 1 & 3 & 1 & 33.0 \\
\hline e14 & 2 & 2013-03-02 & 1.6 & 1.2 & 0.1 & 12.6 \\
\hline e15 & 3 & 2014-04-19 & 0.35 & 0.35 & 0.08 & 4.9 \\
\hline e15 & 2 & 2012-06-21 & 5 & 7 & 1 & 27.0 \\
\hline e16 & 2 & $2012-10-16$ & 0.05 & 0.14 & 0.04 & 5.9 \\
\hline e17 & 2 & $2012-10-16$ & 0.1 & 0.5 & 0.2 & 2.5 \\
\hline e17 & 1 & $1993-03-16$ & - & - & 0.5 & 22.5 \\
\hline e18 & 3 & 2014-04-19 & 0.51 & 0.37 & 0.08 & 4.9 \\
\hline e18 & 2 & 2012-06-21 & -0 & 1 & 1 & 33.0 \\
\hline e19 & 2 & 2013-03-02 & 1.3 & 1 & 0.1 & 12.6 \\
\hline e2 & 1 & $1992-10-25$ & 14 & 14 & 0.2 & 4.9 \\
\hline e2 & 2 & $2012-06-21$ & $1.6 \times 10^{2}$ & $1.7 \times 10^{2}$ & 1 & 27.0 \\
\hline e20 & 3 & 2014-04-19 & 0.26 & 0.38 & 0.05 & 5.9 \\
\hline $\mathrm{e} 21$ & 2 & $2012-10-16$ & 0.5 & 1.4 & 0.2 & 2.5 \\
\hline $\mathrm{e} 21$ & 1 & $1993-03-16$ & 0.4 & 1.1 & 0.5 & 22.5 \\
\hline $\mathrm{e} 22$ & 2 & $2012-10-16$ & 0.12 & 0.33 & 0.05 & 4.9 \\
\hline $\mathrm{e} 22$ & 2 & $2012-06-21$ & 0 & 2 & 1 & 33.0 \\
\hline e23 & 2 & 2013-03-02 & 0.5 & 0.4 & 0.1 & 12.6 \\
\hline e3 & 1 & $1992-10-25$ & 8 & 7.9 & 0.2 & 4.9 \\
\hline e3 & 2 & $2012-06-21$ & 8 & 11 & 1 & 27.0 \\
\hline e4 & 3 & 2014-04-19 & 4.3 & 4.6 & 0.05 & 5.9 \\
\hline e5 & 2 & 2012-10-16 & 0.1 & 4.1 & 0.2 & 2.5 \\
\hline e5 & 1 & $1993-03-16$ & 15 & 17 & 0.5 & 22.5 \\
\hline e6 & 2 & $2012-10-16$ & 2.5 & 2.6 & 0.05 & 4.9 \\
\hline e6 & 2 & 2012-06-21 & 1 & 2 & 1 & 33.0 \\
\hline e7 & 2 & $2013-03-02$ & 0 & 0.2 & 0.1 & 12.6 \\
\hline$e 8 n$ & 1 & $1992-10-25$ & 1.5 & 1.5 & 0.2 & 4.9 \\
\hline e8n & 2 & $2012-06-21$ & 4 & 6 & 1 & 27.0 \\
\hline e8s & 3 & 2014-04-19 & 1.1 & 1.3 & 0.05 & 5.9 \\
\hline e9 & 2 & $2012-10-16$ & 2.6 & 3 & 0.2 & 2.5 \\
\hline e9 & 1 & $1993-03-16$ & 0.3 & 2.4 & 0.5 & 22.5 \\
\hline
\end{tabular}

Notes. An excerpt from the point source catalog. For the full catalog, see Table A.1.

and the nearby fainter PDR to the northeast. The similarity suggests that these features are PDRs, highlighting the sharp interaction points between the $\mathrm{H}$ II region and the surrounding molecular cloud.

The two most prominent of these sharp features are on opposite sides of the W51 Peak (Fig. 4, blue arrows). The long vertical filament on the left (Filament A) approximately faces W51e, while the S-shaped filament on the right (Filament B) faces outward, away from the e1/e2 group and toward a region that contains no known OB stars. The presence of multiple PDR features raises questions about the ionizing source(s). In Orion, the Trapezium is $0.2 \mathrm{pc}$ in projection from the Bar PDR. Filament A 
Table 5. $\mathrm{H}_{2} \mathrm{CO} 2_{1,1}-2_{1,2}$ emission line parameters.

\begin{tabular}{ccccccccc}
\hline \hline Object name & $\begin{array}{c}\text { Amplitude } \\
\text { mJy }\end{array}$ & $E$ (amplitude) $)$ & $\begin{array}{c}V_{\mathrm{LSR}} \\
\mathrm{km} \mathrm{s}^{-1}\end{array}$ & $E\left(V_{\mathrm{LSR}}\right)$ & $\sigma_{V}$ & $E\left(\sigma_{V}\right)$ & $r_{\text {eff }}$ & Detection status \\
\hline NorthCore & 0.652 & 0.018 & 58.877 & 0.074 & 2.375 & 0.074 & 1.8 & - \\
e2-e8 bridge & 0.369 & 0.022 & 56.554 & 0.07 & 1.0 & 0.07 & 1.4 & - \\
e2_a & 0.641 & 0.026 & 57.412 & 0.1 & 2.167 & 0.1 & 0.9 & - \\
e2_b & 1.085 & 0.035 & 56.083 & 0.097 & 2.605 & 0.097 & 0.9 & - \\
e2_c & 0.612 & 0.026 & 56.01 & 0.19 & 3.95 & 0.19 & 0.9 & - \\
e8mol & 1.551 & 0.061 & 60.55 & 0.13 & 2.85 & 0.13 & 0.5 & - \\
e8mol_ext & 1.045 & 0.024 & 60.16 & 0.089 & 3.439 & 0.089 & 1.0 & weak \\
e10mol & 0.999 & 0.039 & 58.01 & 0.21 & 4.69 & 0.21 & 0.5 & - \\
e10mol_ext & 0.813 & 0.018 & 58.507 & 0.094 & 3.593 & 0.094 & 1.1 & weak \\
\hline
\end{tabular}

Notes. Columns with $E$ denote the errors on the measured parameters. $\sigma_{V}$ is the 1-dimensional Gaussian velocity dispersion. $r_{\text {eff }}$ is the effective aperture radius.

Table 6. $\mathrm{H}_{2} \mathrm{CO} 2_{1,1}-2_{1,2}$ absorption line parameters.

\begin{tabular}{ccccccccc}
\hline \hline Object name & $\begin{array}{c}\text { Amplitude } \\
\text { mJy }\end{array}$ & $E$ (amplitude) & $\begin{array}{c}V_{\mathrm{LSR}} \\
\mathrm{km} \mathrm{s}^{-1}\end{array}$ & $E\left(V_{\mathrm{LSR}}\right)$ & $\sigma_{V}$ & $E\left(\sigma_{V}\right)$ & $\begin{array}{c}r_{\text {eff }} \\
{ }^{\prime}\end{array}$ & Detection status \\
\hline e1 & -2.922 & 0.034 & 62.531 & 0.078 & 5.81 & 0.078 & 0.6 & ambig \\
e2 & -21.186 & 0.082 & 56.8728 & 0.009 & 2.0231 & 0.009 & 0.6 & - \\
e3 & -2.57 & 0.1 & 64.26 & 0.11 & 2.35 & 0.11 & 0.4 & - \\
e5 & -1.944 & 0.094 & 62.739 & 0.032 & 0.576 & 0.032 & 0.6 & - \\
e6 & -0.598 & 0.015 & 63.771 & 0.061 & 2.149 & 0.061 & 1.8 & - \\
e9 & -0.477 & 0.078 & 55.4 & 0.22 & 1.15 & 0.22 & 0.5 & - \\
e10 & -0.63 & 0.1 & 66.74 & 0.21 & 1.1 & 0.21 & 0.5 & - \\
\hline
\end{tabular}

Notes. Columns with $E$ denote the errors on the measured parameters. $\sigma_{V}$ is the 1-dimensional Gaussian velocity dispersion. $r_{\text {eff }}$ is the effective aperture radius.

Table 7. H77 $\alpha$ emission line parameters.

\begin{tabular}{cccccccc}
\hline \hline Object name & $\begin{array}{c}\text { Amplitude } \\
\text { mJy }\end{array}$ & $\begin{array}{c}E \text { (amplitude) } \\
\mathrm{mJy}\end{array}$ & $\begin{array}{c}V_{\mathrm{LSR}} \\
\mathrm{km} \mathrm{s}^{-1}\end{array}$ & $\begin{array}{c}E\left(V_{\mathrm{LSR}}\right) \\
\mathrm{km} \mathrm{s}^{-1}\end{array}$ & $\begin{array}{c}\sigma_{V} \\
\mathrm{~km} \mathrm{~s}^{-1}\end{array}$ & $\begin{array}{c}E\left(\sigma_{V}\right) \\
\mathrm{km} \mathrm{s}^{-1}\end{array}$ & Detection status \\
\hline $\mathrm{e} 1$ & 3.0 & 0.2 & 54.87 & 0.85 & 10.9 & 0.85 & - \\
$\mathrm{e} 2$ & 0.6 & 0.13 & 56.0 & 3.8 & 15.3 & 3.8 & - \\
$\mathrm{e} 3$ & 1.48 & 0.33 & 59.8 & 2.7 & 10.5 & 2.7 & - \\
$\mathrm{e} 4$ & 0.56 & 0.39 & 57.2 & 4.4 & 5.4 & 4.4 & - \\
$\mathrm{e} 5$ & 0.25 & 0.18 & 52.6 & 4.7 & 5.8 & 4.7 & weak \\
$\mathrm{e} 6$ & 0.183 & 0.051 & 68.6 & 4.9 & 15.4 & 4.9 & - \\
$\mathrm{e} 9$ & 0.15 & 0.18 & 66.8 & 7.9 & 5.5 & 7.9 & weak \\
$\mathrm{e} 10$ & 0.27 & 0.2 & 51.2 & 9.8 & 11.6 & 9.8 & weak \\
\hline
\end{tabular}

is $0.6 \mathrm{pc}$ from the W51e cluster, which contains enough O-stars to illuminate the filaments, but there may not be a clear lineof-sight from those stars, which appear to be deeply embedded in molecular material, to the filament. These features therefore indicate the presence of additional OB stars that are obscured along our line-of-sight but that are not deeply embedded.

Additionally, the W51 IRS1 ridge is detected in $\mathrm{H} 77 \alpha$. Filament $\mathrm{B}$ peaks in the range $40-50 \mathrm{~km} \mathrm{~s}^{-1}$. Filament $\mathrm{A}$ is the sharp edge feature spanning $v_{\mathrm{LSR}} \sim 38-48 \mathrm{~km} \mathrm{~s}^{-1}$. From Filament $\mathrm{A}$, there is continuous $\mathrm{H} 77 \alpha$ emission toward the northeast from 40 to $80 \mathrm{~km} \mathrm{~s}^{-1}$ that exhibits a gradient from blue to red from southeast to northwest.

The $\mathrm{H} 77 \alpha$ lines reveal two circular features (bubbles) that overlap along the line of sight but are distinct in velocity (Fig. 5). A smaller bubble at $40 \mathrm{~km} \mathrm{~s}^{-1}$ shows a velocity gradient orthogonal to the gradient seen next to filament A, increasing in velocity from southwest to northeast; this gradient may trace the edges of an expanding spherical shell. The large bubble is centered at about $65 \mathrm{~km} \mathrm{~s}^{-1}$ and shows no clear gradients (Fig. 5, for labels see Fig. A.2).

The W51 IRS1 H II region appears connected to the W51 IRS2 bubble in the continuum (Fig. 4 shows the apparentlyconnected region, Fig. 6 shows the IRS2 bubble), but the RRL emission peaks at around $75 \mathrm{~km} \mathrm{~s}^{-1}$ (Fig. 5). Along this region, there are weak "striations" at PA 126 degrees, parallel to the cyan arrow in Fig. 4 and orthogonal to the direction toward IRS2. These striations may trace the outer edge of the expanding IRS2 region. There is also an arc to the southwest of this region that appears to be a bow shock from gas outflowing along the same axis; the cyan arrow points to this arc in Fig. 4.

The IRS2 region peaks in velocity around $62 \mathrm{~km} \mathrm{~s}^{-1}$ at the center, but exhibits a gradient from its center to its surroundings, with a more extended component peaking around $47 \mathrm{~km} \mathrm{~s}^{-1}$. There is a shell around IRS2 with maximum projected radius 


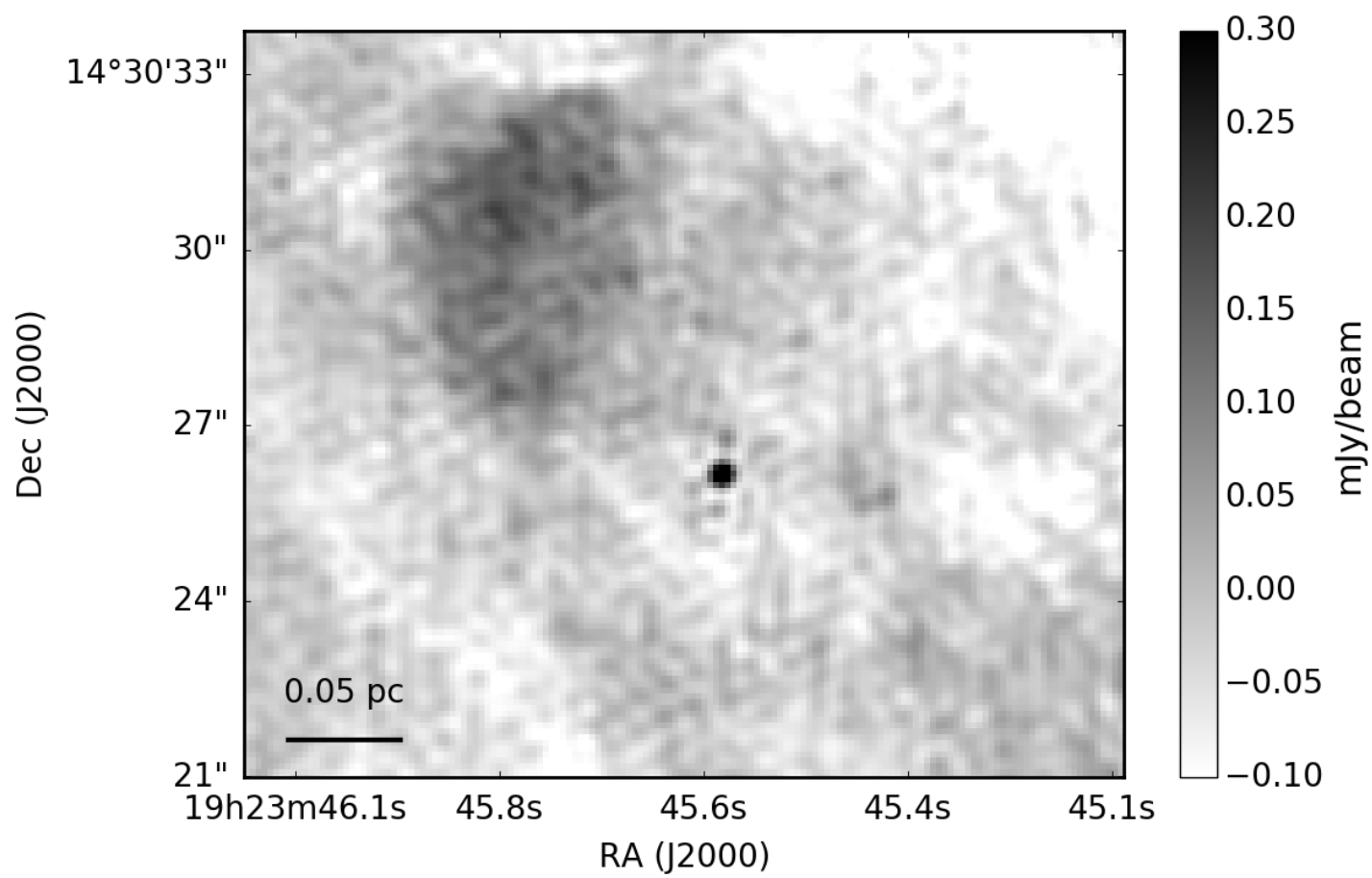

Fig. 3. A bow-shaped feature toward the northeast of the source e11 in the $K \mathrm{u}$-band $(14.5 \mathrm{GHz})$ continuum image. The feature resembles the bow shocks of Herbig-Haro objects, but is more likely to be an H II region given its smooth structure. The synthesized beam size is $\theta_{\text {FwHM }} \approx 0.33^{\prime \prime}$.

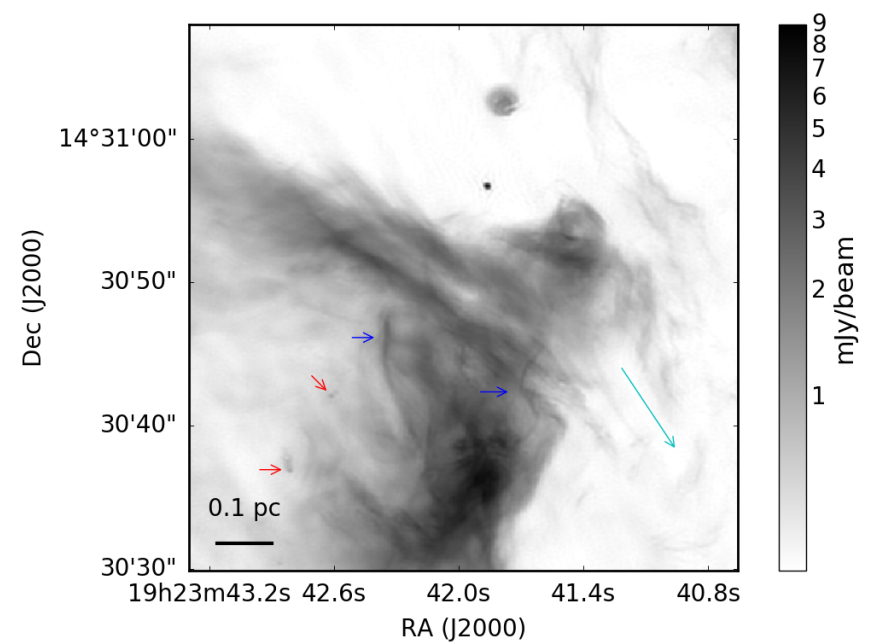

(a)

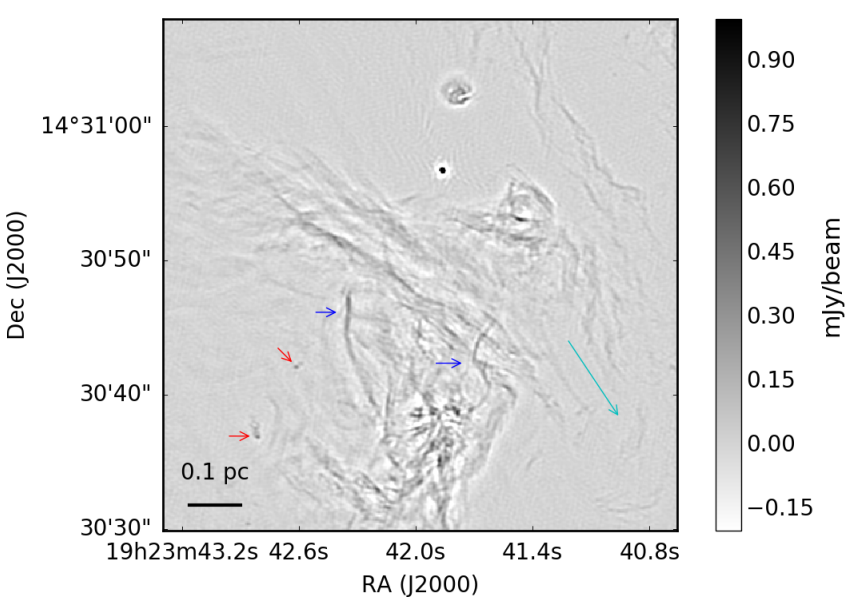

(b)

Fig. 4. a) A C-band (4.9 GHz) image of W51 IRS1, the location of peak intensity on the W51 Main shell. This region accounts for more than half of the radio luminosity of W51. The colorbar has been selected to emphasize the pointlike sources even though it saturates some of the diffuse emission. The synthesized beam size is $\theta_{\text {FWHM }} \approx 0.3^{\prime \prime}$. b) An unsharp-masked version of the image with a $0.3^{\prime \prime}$ smoothing kernel used for the large-angular-scale flux removal. There are two pointlike sources in field marked by red arrows, e14 at center-left and the cometary e13 toward the lower left. The filamentary features mentioned in Sect. 3.4 are identified by blue arrows. The cyan arrow points along a possible ionized flow, traced by elongated features parallel to the arrow and a rounded feature to the southwest. A counterpart to the northeast is barely visible.

$21^{\prime \prime}(0.5$ pc; Figs. 6 and 7). A prominent sharp edge feature (blue arrow in Fig. 6) extends to the northeast from the central cluster; it may trace the outflow of hot material from the cluster.

Parts of the shell close to IRS2 are detected in radio recombination lines $(\mathrm{H} 77 \alpha)$, but the more distant regions marked in Fig. 6 with red arrows are not (they are too faint). The shell to the northwest peaks at $v_{\mathrm{lsr}}=37.5 \mathrm{~km} \mathrm{~s}^{-1}$, and a gradient is observed from that shell to the peak velocity of IRS2 at $v_{\text {lsr }}=62.6 \mathrm{~km} \mathrm{~s}^{-1}$. The velocity structure is inconsistent with spherical outflow but is consistent with a conical flow structure. This flow structure likely indicates that the cluster has evacuated a large cavity approximately toward the observer, consistent with the low observed extinction toward IRS2.

\subsection{The Lacy IRS2 ionized outflow}

Lacy et al. (2007) reported the detection of very high-velocity ionized gas in the mid-infrared [Ne II] $12.8 \mu \mathrm{m}$ and S IV $10.5 \mu \mathrm{m}$ lines. They observed the gas at a velocity blueshifted about $100 \mathrm{~km} \mathrm{~s}^{-1}$ from the IRS2 ionized and molecular gas velocity. 

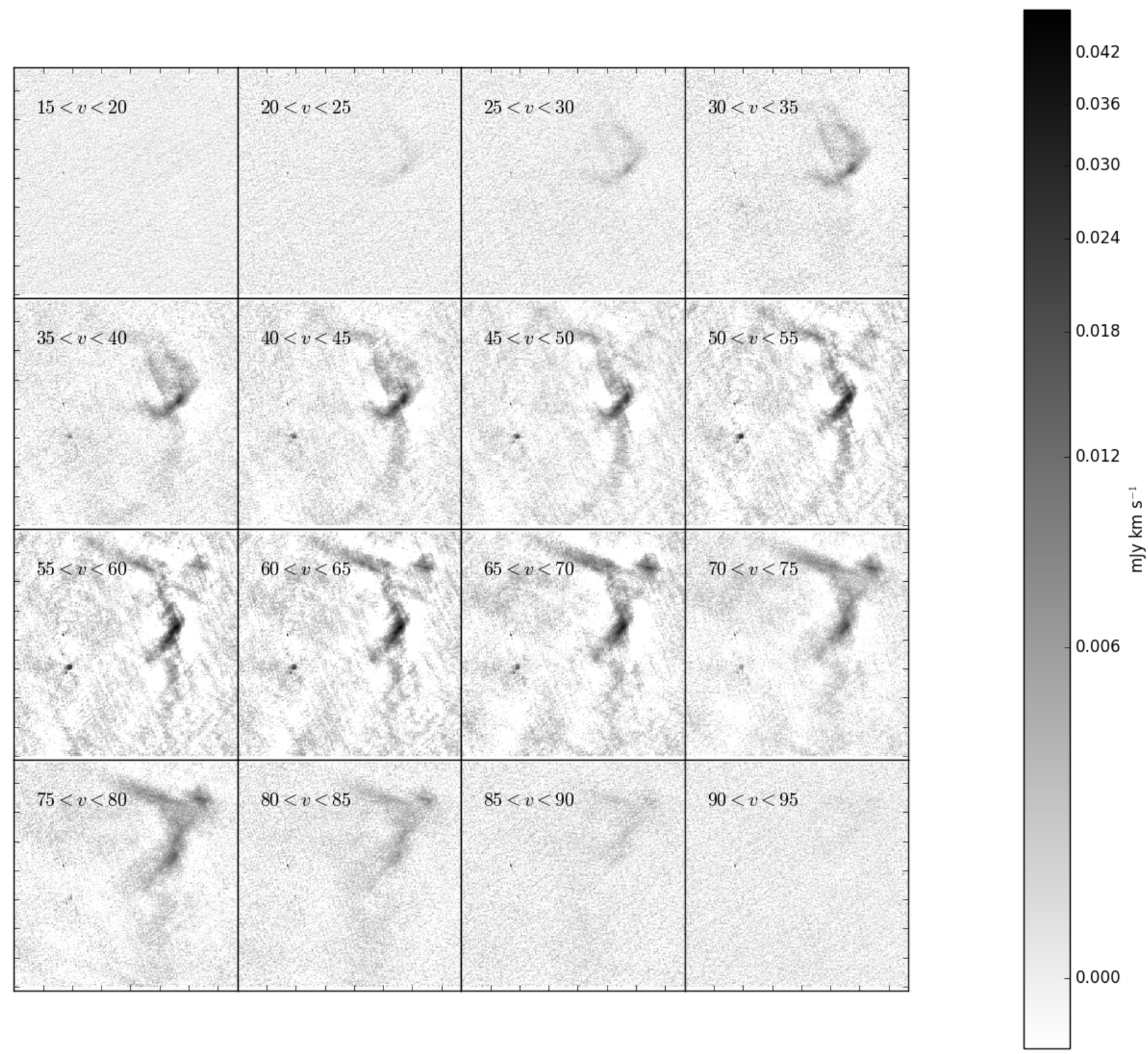

Fig. 5. Channel maps of the H77 $\alpha$ radio recombination line integrated over $5 \mathrm{~km} \mathrm{~s}^{-1}$ windows for W51 Main and IRS1. There are small ring features notable at low and high velocities; these are identified in Fig. A.2. These images demonstrate that there are two distinct but possibly interacting bubbles along the line of sight in W51 Main. The synthesized beam size is $\theta_{\text {FWHM }} \approx 0.42^{\prime \prime}$.

We have detected the same feature in the H77 $\alpha$ RRL. The RRL shows the same position-velocity structure as the infrared ionized features. No redshifted counterflow is detected (Fig. 8).

One minor but notable feature of the $\mathrm{H} 77 \alpha$ flow is that the emission occurs close to the rest velocity of the He77 $\alpha$ line. If Lacy et al. (2007) had not already unambiguously identified the high-velocity of this feature in two infrared lines, we would have assumed the detected emission to be slightly redshifted Helium, since $\mathrm{He} 77 \alpha$ is detected at $v \sim 60 \mathrm{~km} \mathrm{~s}^{-1}$ in IRS2, rather than correctly identifying it as $\mathrm{H} 77 \alpha$.

\section{Analysis}

We use the data to examine the following properties of W51:

- Section 4.1: determination of the total stellar mass in W51 based on its infrared luminosity and a brief examination of the probable locations of the massive stars.
- Section 4.2: determination of the object type (e.g., stellar wind, photoionized HII region) associated with each detected continuum source.

- Section 4.2.1: a brief discussion of the compact sources near W51 Main and their role in ionizing the W51 Main bubbles.

- Section 4.2.2: a brief discussion of the IRS2 faint point sources.

- Section 4.3: examination of the o- $\mathrm{H}_{2} \mathrm{CO} \quad 2_{1,1}-2_{1,2^{-}}$ detected hot cores, including upper limits on $4.829 \mathrm{GHz}$ o- $\mathrm{H}_{2} \mathrm{CO} 1_{1,0}-1_{1,1}$ emission.

- Section 4.4: discussion of the $\mathrm{d} 4$ variable emission feature, concluding that it arises from a fast outflow interacting with the dense local ISM.

- Section 4.5: measurements of the velocity dispersion within the $\mathrm{e} 1$ and e 2 clusters based on their RRL and $\mathrm{H}_{2} \mathrm{CO}$ emission velocities, and a derivation of the gas-to-stellar mass ratio on this basis. 


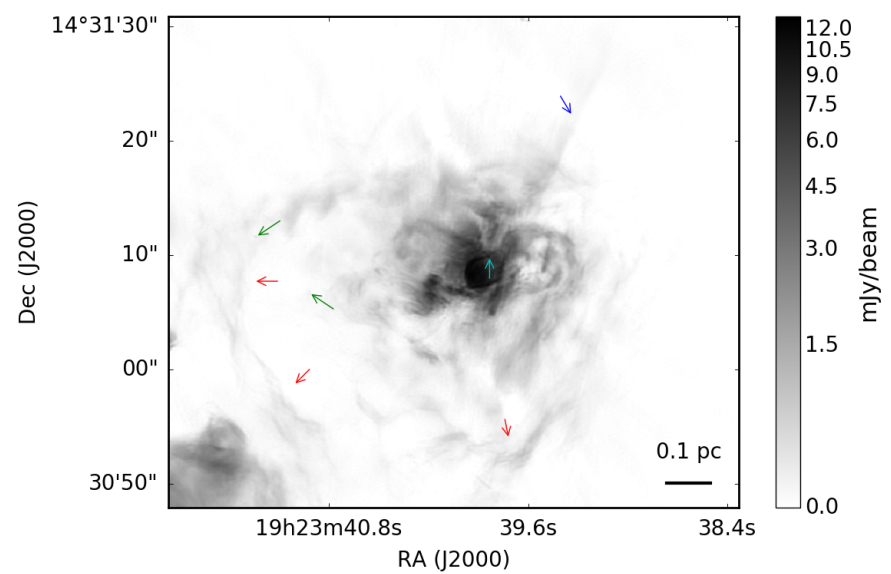

(a)

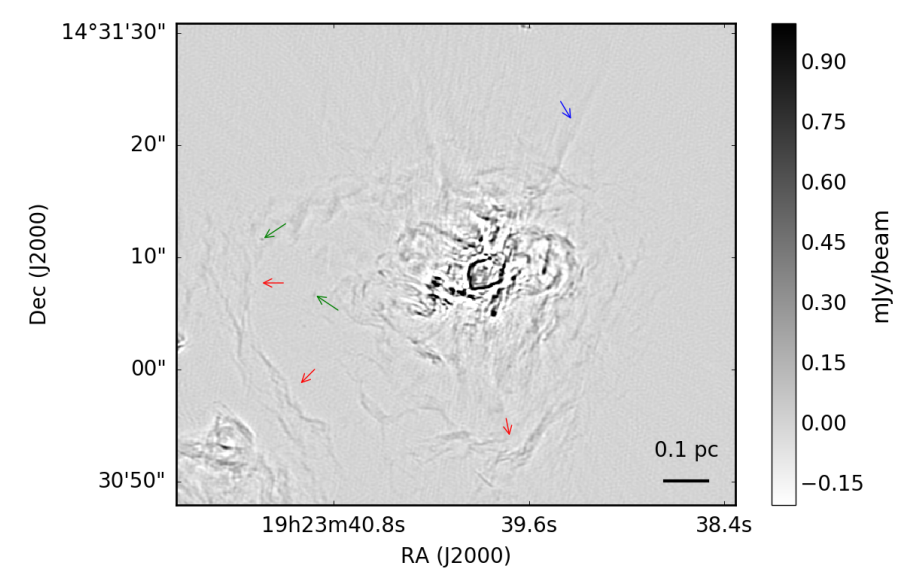

(b)

Fig. 6. a) The W51 IRS2 region in $C$-band continuum and b) unsharp masked with a $0.3^{\prime \prime}$ kernel. Concentric ring features surround the IRS2 region. A V-shaped feature to the north (cyan arrow) may highlight the edge of gas flows out of the cluster, extending to the long linear feature pointing northwest (blue arrow). An apparent shell is observed centered on the IRS2 cluster, with edge features to the south and east (red arrows). The compact sources $\mathrm{d} 6$ and $\mathrm{d} 7$, which are candidate colliding-wind binaries (Sect. 4.2), are identified with green arrows.

- Section 4.6: constraints on the mass loss rate of the relatively low-extinction IRS2 region using RRL emission to constrain the mass and velocity of escaping material.

\subsection{The FIR-deduced stellar mass}

We have re-measured the infrared luminosity of the W51 protoclusters using Herschel Hi-Gal data (Molinari et al. 2010, 2016; Traficante et al. 2011), fitting an SED from the 70 to $500 \mu \mathrm{m}$ with a single blackbody component. While a single-component fit provides a poor measurement of the dust temperature - multiple temperature components are evident (Sievers et al. 1991) - it provides a good approximation to the total infrared luminosity, which is dominated by a single warm $(\sim 60 \mathrm{~K})$ component. The infrared luminosity is about $L \sim 2 \times 10^{7} L_{\odot}$ within a 2 pc radius ${ }^{4}$, which includes both the W51 IRS2 and W51 Main protoclusters. The luminosity estimate does not include the mid-infrared luminosity, which may provide an additional $\sim 25-50 \%$ based on the IRAS 12 and $25 \mu \mathrm{m}$ measurements. Our measured total luminosity is somewhat larger than the previous estimates based on IRAS and KAO measurements (Harvey et al. 1986; Sievers et al. 1991). A luminosity $L=2.0 \pm 0.5 \times 10^{7} L_{\odot}$ implies a stellar mass $M_{c l}=6.7 \pm 2.3 \times 10^{3} M_{\odot}$, with a corresponding number of O-stars (greater than $20 M_{\odot}$ ) $N_{\mathrm{O}}=19 \pm 6$, assuming a Kroupa (2001) IMF at the zero-age main sequence using Vacca et al. (1996) stellar parameters. This estimate provides a lower limit on the present day O-star population, since some of the short-wavelength radiation (i.e., the NIR radiation) is able to escape rather than being reprocessed into the far infrared, which we have used to infer the luminosity.

Of these expected $\sim 20$ O-stars, six have been detected in the near infrared and spectroscopically confirmed. Figuerêdo et al. (2008) found 4 exposed O-type stars and Barbosa et al. (2008) found an additional two with strong infrared excess. None of these coincide with ultracompact or hypercompact H II regions, but all are in the bright and diffuse IRS2 H II region.

Mehringer (1994) found an additional eight ultracompact and hypercompact $\mathrm{H}$ II regions, all of which appear to be B0

\footnotetext{
4 In many bands, W51 Main and W51 IRS2 are saturated, so we have interpolated from neighboring pixels to estimate the flux density. Our luminosity estimate is therefore a lower limit.
}

or earlier stars based on their radio-derived ionizing photon luminosity. The other $\sim 10-20$ O-stars expected to have already formed given the observed total luminosity may be the hypercompact H II regions we have detected. However, it is difficult to explain the total luminosity of the region from hypercompact $\mathrm{H}$ II regions alone, since their emission is necessarily confined by optical depth to a small region, and they contribute minimally to the overall radio luminosity. The thermal radiation from the OBstars in the diffuse H II regions may illuminate a large portion of the cloud and contribute substantially to the infrared luminosity.

It is possible that the O-stars providing most of the observed reprocessed luminosity are near or within the "shell" structure of the W51 IRS1 region (Fig. A.2, Sect. 3.4). This region dominates the optical and radio luminosity of W51 Main (though it provides $\lesssim 1 / 4$ of the FIR luminosity) and is bright enough in near-infrared and radio continuum emission to make detection of point sources impossible, explaining why no O-stars have been previously confirmed. There are multiple overlapping H II bubbles in the W51 Main shell at different velocities (Fig. 5), suggesting that there are interacting and expanding bubbles, which in turn implies that there is a separation between the driving sources and the HII region of at least the bubble sizes, $0.2-$ $0.3 \mathrm{pc}$. This scenario suggests the existence of a population of un-embedded O-stars spread between the W51 protoclusters.

\subsection{The nature of the continuum sources}

Out of the 27 compact continuum sources reported, the majority that we could identify are ultra- or hyper-compact H II regions. The ultracompact $\mathrm{H}$ II regions are identified as resolved sources, with $r>0.005 \mathrm{pc}^{5}$. The HCH II regions are more challenging to identify, as they are observed only as unresolved sources. Normally, these could be identified from their SEDs, which should follow an $\alpha=2$ Rayleigh-Jeans law up to some turnover point, above which they become optically thin and turn over to an $\alpha=-0.1$ power law (Wilson et al. 2009). However,

\footnotetext{
5 The Kurtz (2002) definition of HCH II regions is $r_{\mathrm{H}_{I}}<0.05 \mathrm{pc}$. We detect a few sources that are on the borderline between HCH II and UCH II regions, with $r \sim 0.05 \mathrm{pc}$, and therefore we have identified all unresolved sources at the highest resolution $(0.005 \mathrm{pc})$ as $\mathrm{HCH}$ II regions, and all that are well-resolved are classified as UCH II regions.
} 

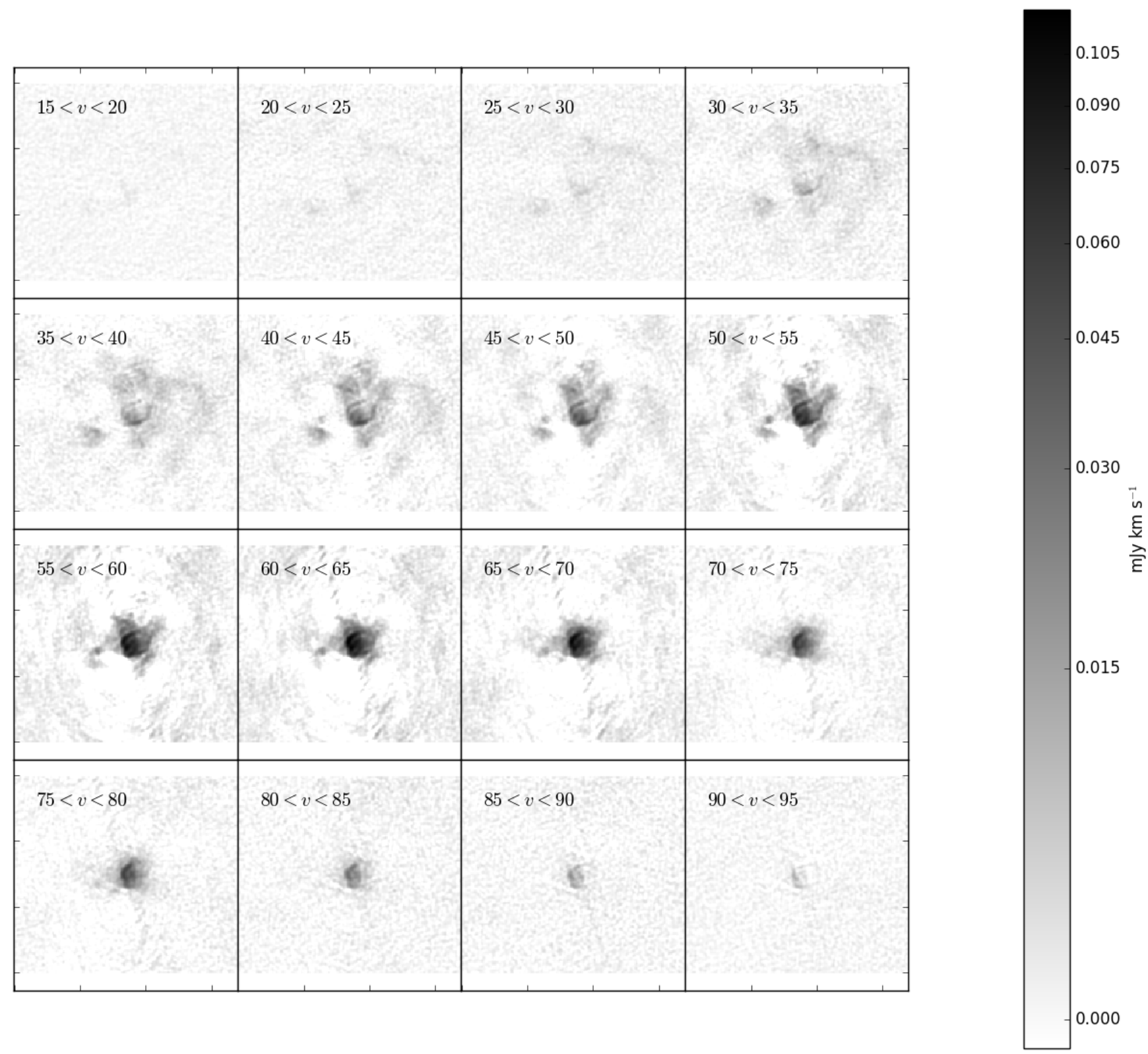

Fig. 7. Channel maps of the $\mathrm{H} 77 \alpha$ line integrated over $5 \mathrm{~km} \mathrm{~s}^{-1}$ windows for the IRS 2 region. Around $30-35 \mathrm{~km} \mathrm{~s}^{-1}$, the edges of the IRS 2 region become visible, illustrating the blueshifted cavity discussed in Sect. 3.4. These images demonstrate the presence of outflowing material and are used in Sect. 3.4 to estimate the ionized gas mass loss rate from IRS2. The synthesized beam size is $\theta_{F W H M} \approx 0.42^{\prime \prime}$.

the SEDs we observed do not exhibit such unambiguous behavior for any cases except e2. For the rest, we see $\alpha \approx 1$ power laws at low frequencies (e.g., e4), negative power-laws at low frequencies (e.g., e9), or entirely flat SEDs (e.g., e10). SED classifications for other sources are given in Table 3, and plots of the SEDs can be seen in Appendix B.

An SED with $\alpha \sim 1$ or $\alpha \sim 0$ can be explained by density gradients in the H II region (Keto et al. 2008; Galván-Madrid et al. 2009; Tanaka et al. 2016). However, a minimum at $~ 6 \mathrm{GHz}$ cannot. Additionally, an optically thick H II region that is just unresolved would have $S_{5 \mathrm{GHz}} \sim 70 \mathrm{mJy}$, so for the sources discussed in this section with $S_{5 \mathrm{GHz}}<1 \mathrm{mJy}$, the upper limit on the source radius of an optically thick $\mathrm{H}$ II region with electron temperature $T_{\mathrm{e}}=10^{4} \mathrm{~K}$ is $\lesssim 160 \mathrm{au}$. If any of these sources are $\mathrm{H}$ II regions, then, they are among the most compact of $\mathrm{HCH}$ II regions in the galaxy, and by extension must be extremely young or accretiondominated (Keto 2003; Peters et al. 2010b). It is unlikely to find a large population of very young O-stars at exactly coincident evolutionary stages distributed across a parsec-scale star-forming region, especially when the clustered groups of compact H II regions exhibit a significant size distribution from $r<0.005 \mathrm{pc}$ to $r \sim 0.05$ pc. Additionally, most of the detected sources are not correlated with regions of high molecular gas density: they do not have a significant reservoir from which to accrete. We therefore discard the hypothesis that the faint sources are all extreme $\mathrm{HCH}$ II regions.

Negative spectral indices at low frequencies are usually assumed to indicate synchrotron emission (Wilson et al. 2009; Condon \& Ransom 2007), but young and forming stars and H II regions are generally not strong synchrotron emitters. However, colliding-wind binaries (CWBs) often exhibit nonthermal SEDs, likely caused by accelerated particles in the windwind collision zone (De Becker \& Raucq 2013). Stellar winds around massive stars may also exhibit $\alpha<2$ power-laws, but 


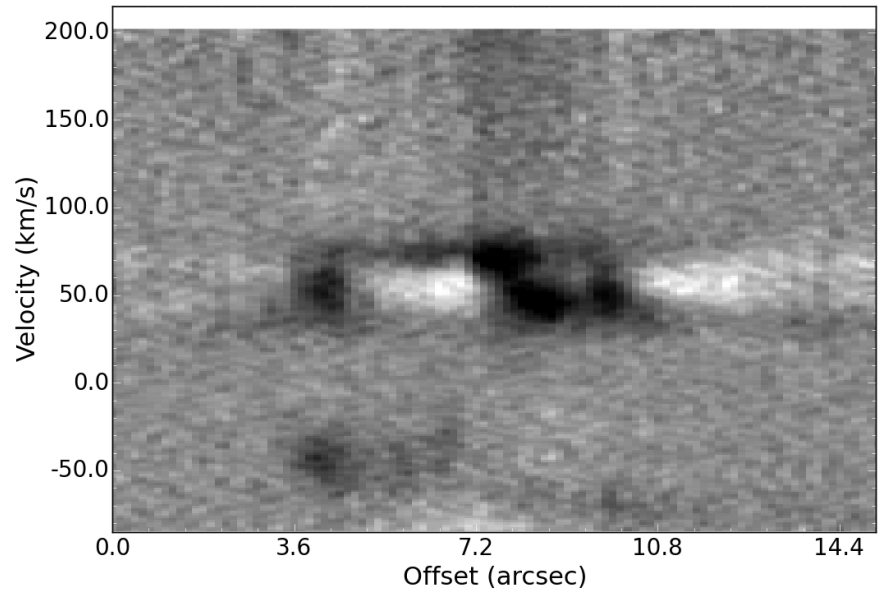

(a)

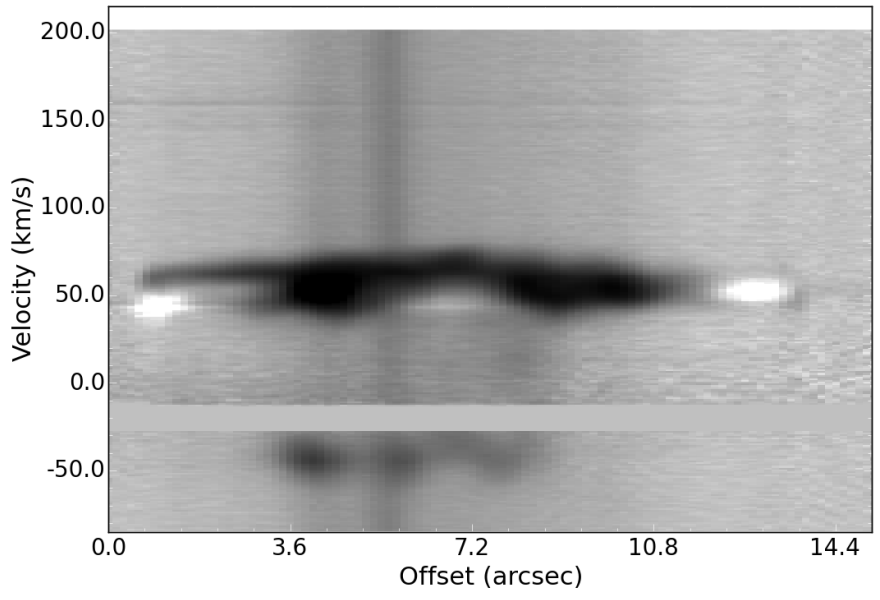

(b)

Fig. 8. Position-velocity slices through a) the H77 $\alpha$ cube and b) the [Ne II] cube from Lacy et al. (2007) tracking the approximate path of the Lacy jet. Emission is black, absorption (which in both images is due to reduction artifacts rather than true physical absorption) is white. The vertical streaks are continuum sources. The abscissa shows the offset position along the slice from J2000 19:23:40.464 +14:31:07.9536 to 19:23:39.4176 $+14: 31: 04.7172$, position angle $258^{\circ}$. The blueshifted lobe is evident at $-50 \mathrm{~km} \mathrm{~s}^{-1}$ in both cubes, and neither shows a redshifted counterpart. The $\mathrm{H} 77 \alpha$ data reveal that there is truly no counterpart, ruling out the possibility that it was simply too extincted to be observed in the infrared.

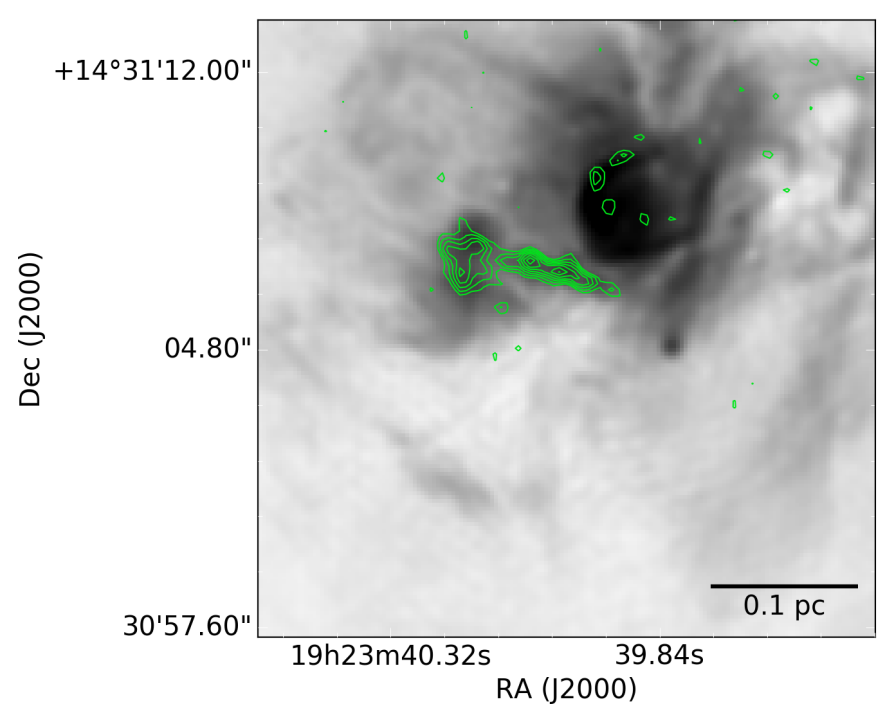

(a)

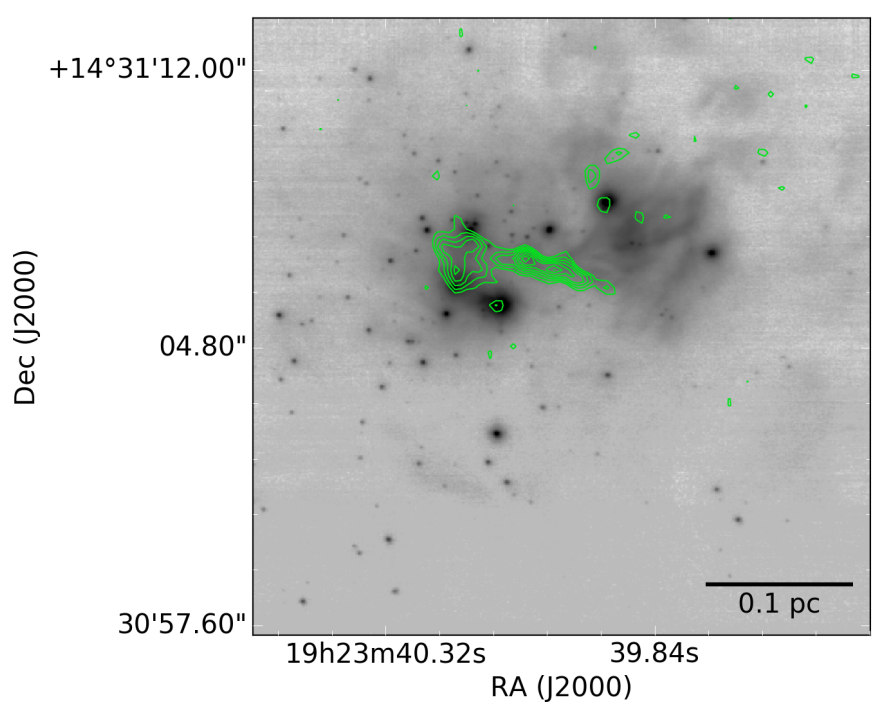

(b)

Fig. 9. H77 $\alpha$ contours of the "Lacy Jet" overlaid on a) the $15 \mathrm{GHz}$ continuum image (synthesized beam $\theta_{\mathrm{FWHM}} \approx 0.33^{\prime \prime}$ ) and b) the NACO $K$-band image (resolution $\approx 0.2^{\prime \prime}$ Barbosa et al. 2008). The H77 $\alpha$ contours are at $0.1,0.2,0.3,0.4,0.5,0.6$, and $0.7 \mathrm{Jy} \mathrm{km} \mathrm{s}{ }^{-1}$, integrated over the velocity range -60 to $-16 \mathrm{~km} \mathrm{~s}^{-1}$.

they are not expected to deviate far from $\alpha \sim 0.6-0.7$ (Wright $\&$ Barlow 1975; Panagia \& Felli 1975; Reynolds 1986).

Radio jets are sometimes observed to have negative spectral indices (Moscadelli et al. 2013, 2016), and they are often faint at distances comparable to W51, making them plausible candidates for the observed faint emission. However, previous radio jets have been identified by their association with $\mathrm{CH}_{3} \mathrm{OH}$ or $\mathrm{H}_{2} \mathrm{O}$ masers, and the faint continuum sources we discuss here do not have any associated masers. Absent any signs of ongoing accretion, jets are not the most likely source of the observed emission, but the current data cannot definitively rule them out.

CWBs typically have radio luminosities $L_{\mathrm{rad}} \sim$ $10^{29}-10^{30} \mathrm{erg} \mathrm{s}^{-1}$ (De Becker \& Raucq 2013). At the distance of W51, this corresponds to $0.5-5 \mathrm{mJy}$ at $5 \mathrm{GHz}$, so such binaries should be detected. By contrast, single star winds range from $L_{\mathrm{rad}} \sim 10^{27}-10^{29} \mathrm{erg} \mathrm{s}^{-1}$, with only a few known in the high range (Bieging et al. 1989), so these are less likely to be detected.

Both CWBs and radio-bright stellar wind sources should be bright in the optical and near-infrared, with stellar luminosities $10^{5}-10^{6} L_{\odot}$. Much of the W51 protocluster region is obscured in the infrared by extinction from the Galactic plane and the W51 GMC, but in the near-IR an unextincted O9 star would have $m_{K} \sim 10.5$ (Pecaut \& Mamajek 2013) ${ }^{6}$, or $m_{K} \sim 13.0$ with Galactic disk line-of-sight extinction $A_{K}=2.6$ (Goldader $\&$ Wynn-Williams 1994). Some of these stars therefore ought to be detected in the NIR; we report such detections in Table 8.

6 http://www . pas.rochester.edu/ emamajek/EEM_dwarf UBVIJHK_colors_Teff.txt 


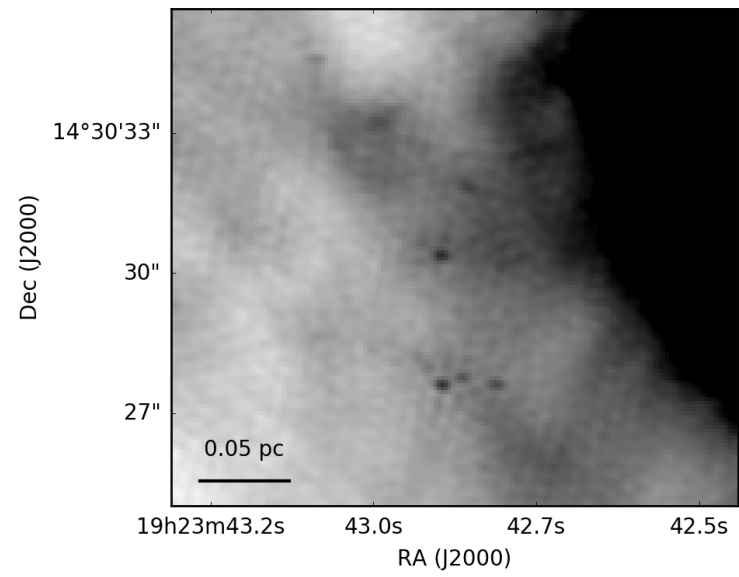

(a)
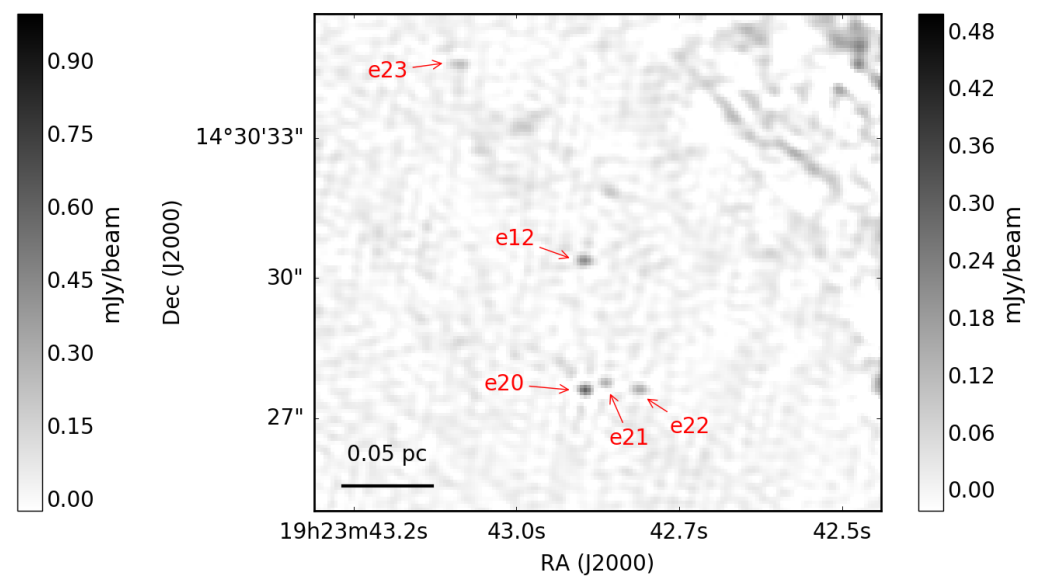

(b)

Fig. 10. a) A $C$-band image of the W51e20/e21/e22 cluster. b) An unsharp-masked version of the image with a 0.3" smoothing kernel. Sources e20, e21, and e22 correspond to two detected infrared $K$-band sources from Goldader \& Wynn-Williams (1994, Table 8) and have $K$-band luminosities consistent with spectral type $\mathrm{O} 4 \mathrm{~V}$ or earlier. The other point sources seen in this field are not detected in the near infrared.

Table 8. Source associations.

\begin{tabular}{llll}
\hline \hline Source name & X-ray & NIR & Goldader 1994 \\
& & & \\
\hline $\mathrm{d} 3$ & - & UGPSJ192335.88+143128.8 & - \\
$\mathrm{d} 4 \mathrm{e}$ & CXOJ192339.6+143130 & UGPSJ192339.65+143130.9 & - \\
$\mathrm{d} 4 \mathrm{w}$ & $($ same as d4e $)$ & (same as d4e) & - \\
$\mathrm{d} 5$ & - & UGPSJ192341.77+143127.6 & - \\
$\mathrm{d} 6$ & CXOJ192341.1+143110 & UGPSJ192341.29+143111.8 & - \\
$\mathrm{d} 7$ & CXOU192340.96+143106.7 & UGPSJ192340.91+143106.7 & RS15 \\
e7 & - & UGPSJ192344.79+142911.2 & - \\
e14 & - & UGPSJ192342.60+143042.2 & - \\
e15 & - & UGPSJ192338.65+143005.8 & - \\
e20 & CXOU192342.86+143027.5 & UGPSJ192342.85+143027.7 & RS7 \\
e21 & (same as e20) & (same as e20) & (same as e20) \\
e22 & CXOU192342.77+143027.5 & UGPSJ192342.84+143027.5 & RS8 \\
\hline
\end{tabular}

Based on these arguments, we have classified each detected compact source in Table 3 . We report 12 candidate CWBs throughout the observed region. These sources constitute a large fraction of the un-embedded O-star population discussed in Sect. 4.1, and their presence confirms that un-embedded, mainsequence O-stars reside in the same cloud as the still-forming clusters.

\subsubsection{The faint radio continuum sources in W51 Main}

The sources e20, e21, and e22 are likely members of a distributed O-star population, rather than members of the clusters of compact H II regions (Fig. 10). They were detected by Goldader \& Wynn-Williams (1994) in the $K$-band at $m_{K}<12$, making these the brightest NIR sources outside of W51 IRS2 (Table 8 gives their associated NIR source names from large surveys). Their luminosities suggest they are O-type stars. Since they were also detected in X-rays (Townsley et al. 2014), these are strong candidate CWBs. The high brightness in the infrared $K$-band and weak radio continuum from these sources, in contrast with the bright radio continuum and absence of infrared emission from e1/e2, supports this hypothesis. Assuming they are early O-stars, e20, e21, and e22 are capable of providing the ionizing and infrared luminosity observed from the W51 IRS1 H II region.
Some questions about the continuum sources remain unanswered: how close are the e1/e2 and e20/e21/e22 clusters? They are separated by $0.36 \mathrm{pc}$ in projection, but there is no direct information about the line-of-sight velocity of the e20 cluster. The other sources in this general area that do not have infrared counterparts, e12, e13, and e23, are also likely OB stars behind infrared dark clouds that obscure them in the near infrared. It is possible that all of the W51 IRS1 and W51 Main compact sources are within a common $\sim 0.5 \mathrm{pc}$ sphere.

\subsubsection{The faint radio continuum sources in W51 IRS2}

Of the compact radio sources identified near W51 IRS2, all but d2 have infrared associations (Table 8). Four of them also have X-ray associations. Except for d3, which is associated with a moderately extended $\mathrm{H}$ II region, these are candidate CWBs.

An alternate explanation for the SED, location, and variability of $\mathrm{d} 4$ is discussed in Sect. 4.4.

\section{3. $\mathrm{H}_{2} \mathrm{CO}$ emission features}

$\mathrm{H}_{2} \mathrm{CO}$ is generally a good tracer of molecular gas, showing up at typical abundances $\sim 10^{-9} \mathrm{H}_{2}$ wherever $\mathrm{CO}$ is detected (e.g., Mangum \& Wootten 1993). The centimeter lines of o- $\mathrm{H}_{2} \mathrm{CO}$ are 


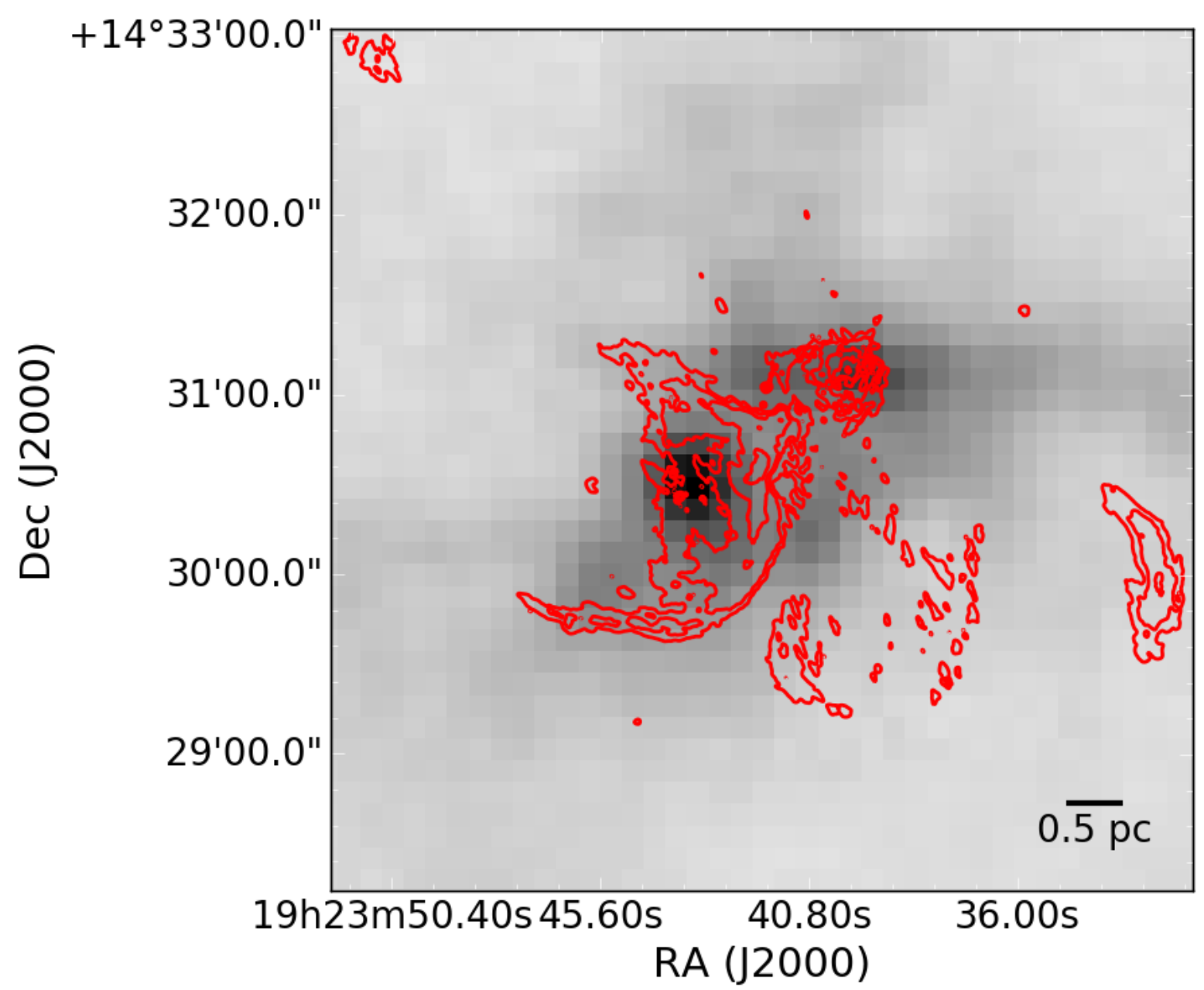

Fig. 11. Contours of the $C$-band $\left(4.9 \mathrm{GHz}\right.$ ) continuum overlaid on an integrated intensity map of $\mathrm{C}^{18} \mathrm{O} 3-2$ from 45 to $65 \mathrm{~km} \mathrm{~s}^{-1}$ (Parsons et al. 2012). The contours go from 0.1 to $10 \mathrm{mJy}$ in 5 logarithmic steps. The $\mathrm{C}^{18} \mathrm{O}$ peaks on the e1/e 2 region and has a clear minimum corresponding to the peak of the radio continuum emission.

often used to measure gas density when detected in absorption (e.g., Ginsburg et al. 2011; Zeiger \& Darling 2010). o- $\mathrm{H}_{2} \mathrm{CO}$ $1_{1,0}-1_{1,1}$ and $2_{1,1}-2_{1,2}$ are commonly observed in absorption but rarely in emission (e.g., Mangum \& Wootten 1993; Araya et al. 2007). The $2_{1,1}-2_{1,2}$ line has been observed in emission in the starburst M82 (Mangum et al. 2008), behind the Orion nebula (Evans et al. 1975; Kutner et al. 1976; Batrla et al. 1983; Johnston et al. 1983; Bastien et al. 1985; Wilson \& Johnston 1989), in $\rho$ Ophiucus B (Loren et al. 1980, 1983; Martin-Pintado et al. 1983; Wadiak et al. 1985), and in DR 21 (Wilson et al. 1982; Johnston et al. 1984). However, the $2_{1,1}-2_{1,2}$ line has so far only been observed in absorption at large distances $(d>2$ kpc) within the Galaxy.

We have detected three regions of $2_{1,1}-2_{1,2}$ emission in the W51 region, all corresponding to previously detected hot molecular cores (Zhang \& Ho 1997; Shi et al. 2010b,a; Goddi et al. 2015, 2016). W51e2 is partially surrounded by a "halo" of $\mathrm{H}_{2} \mathrm{CO} 2_{1,1}-2_{1,2}$ emission to its northeast; the $\mathrm{HCH}$ II region itself shows only $2_{1,1}-2_{1,2}$ absorption because the continuum source is bright (Figs. 13 and 15). The $\mathrm{H}_{2} \mathrm{CO}$ emission corresponds to the cores W51e2e and W51e2nw and the diffuse emission between them (Goddi et al. 2016; Shi et al. 2010b). The HCH II region W51e8 exhibits extended $2_{1,1}-2_{1,2}$ emission, including a somewhat diffuse region between W51e4 and W51e1. Finally, in W51 IRS2, there is extended $2_{1,1}-2_{1,2}$ emission between W51d1 and W51d2, adjacent to the $\mathrm{NH}_{3}$ masers observed by Zhang \& Ho (1995) and more recently Goddi et al. (2015), and aligned with the Zapata et al. (2010) W51 North Disk and thermal $\mathrm{NH}_{3}$ emission (Fig. 12).

In all cases, the emission is extended and spread smoothly over multiple velocity channels (Figs. 13, 16, 17). It is therefore not maser emission.

None of these regions are detected in $1_{1,0}-1_{1,1}$ emission in our data. These nondetections are likely because our brightness sensitivity at $C$-band $(4.9 \mathrm{GHz})$ is poor. The upper limit is $T_{B}<300 \mathrm{~K}$ (Table 2), ruling out the presence of strong masers. Additionally, the foreground cloud is a much stronger absorber in the $1_{1,0}-1_{1,1}$ than in the $2_{1,1}-2_{1,2}$ line, so any emission is likely to be obscured by foreground absorption.

The W51e2, W51e8, and North cores share some properties but are not identical. All three exhibit peak $\mathrm{H}_{2} \mathrm{CO} 2_{1,1}-2_{1,2}$ brightness temperatures $T \sim 30-60 \mathrm{~K}$ at $14.5 \mathrm{GHz}$. W51e8 has a brightness temperature and spatial extent similar to that of W51 North, though it is also connected with the e10 molecular emission and is morphologically more complex. W51e8 contains a hypercompact H II region, while W51 North does not. However, in W51 North, detection of a hypercompact H II region may be prevented by confusion with the nearby bright IRS2: it is therefore plausible that W51 North contains a similar continuum source to W51e8 ( $\left.S_{v} \sim 1 \mathrm{mJy}\right)$. Unlike W51e8 and North, 


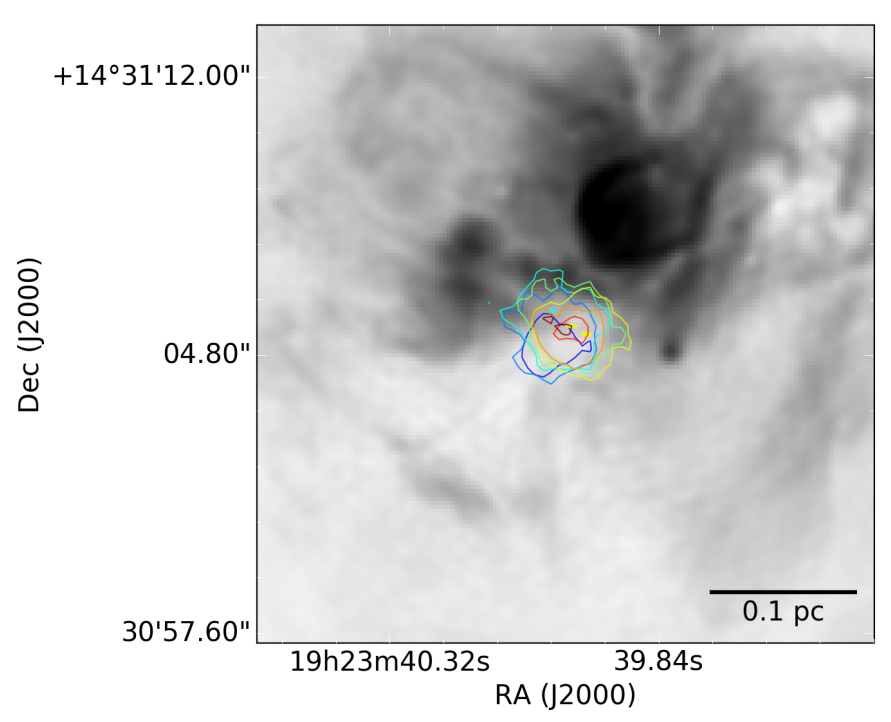

(a)

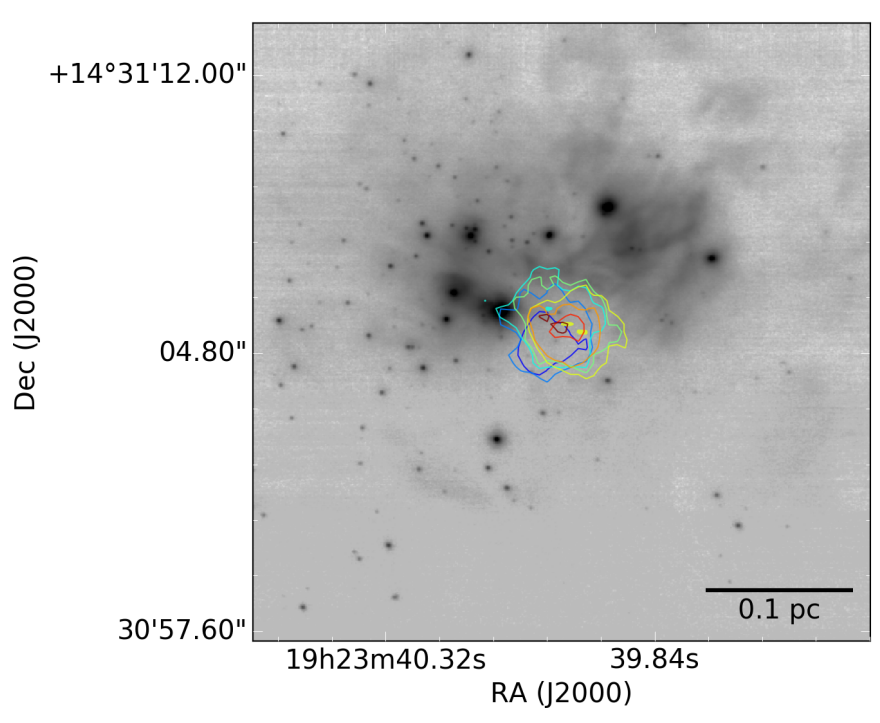

(b)

Fig. 12. $\mathrm{H}_{2} \mathrm{CO} 2_{1,1}-2_{1,2}$ emission contours of the W51 North core overlaid on a) the $14 \mathrm{GHz}$ continuum and b) the NACO $K$-band continuum image (Barbosa et al. 2008). The contours are at $3 \mathrm{mJy} / \mathrm{beam}$ in each channel with width $0.5 \mathrm{~km} \mathrm{~s}^{-1}$ at velocities 56 (blue) to 60 (red) $\mathrm{km} \mathrm{s}^{-1}$.

W51e2 has a more extended morphology including the cores W51e2e and W51e2nw, and it includes the bright $\mathrm{HCH}$ II region e2w (Shi et al. 2010b; Goddi et al. 2016).

We estimate the mass of the W51 North core in a few ways. A reasonable lower limit on the mass is given by assuming it has a volume density $n \gtrsim 10^{6} \mathrm{~cm}^{-3}$, which is required to detect it in emission in $\mathrm{o}-\mathrm{H}_{2} \mathrm{CO} 2_{1,1}-2_{1,2}$ (Mangum \& Wootten 1993). The core radius, as measured by fitting a $2 \mathrm{D}$ Gaussian to its integrated intensity emission map, is $\sigma=0.9^{\prime \prime}$ or $0.025 \mathrm{pc}$ $\left(F W H M=2.1^{\prime \prime}, 0.056 \mathrm{pc}\right)$. If we assume the core is spherically symmetric, the resulting mass is $M \gtrsim 14 M_{\odot}$. We compute the o- $\mathrm{H}_{2} \mathrm{CO} 2_{1,1}-2_{1,2}$ column density using Mangum \& Shirley (2015) equation 100, with a measured integrated intensity of $55 \mathrm{mJy} \mathrm{km} \mathrm{s}{ }^{-1}$ or $65 \mathrm{~K} \mathrm{~km} \mathrm{~s}^{-1}$ given the $2 \times 1.5^{\prime \prime}$ source area. The inferred column of o- $\mathrm{H}_{2} \mathrm{CO}$, assuming LTE, is $\sim 5 \times 10^{15}-5 \times 10^{16} \mathrm{~cm}^{-2}$, depending on the assumed kinetic temperature and excitation temperature (20-200 K). This $\mathrm{H}_{2} \mathrm{CO}$ column implies an implausibly large mass $\left(M \sim 5 \times 10^{3} M_{\odot}\right)$ for typical assumed abundances $\left(X_{\mathrm{H}_{2} \mathrm{CO}} \sim 10^{-9}\right)$, so we conclude that the $\mathrm{H}_{2} \mathrm{CO}$ abundance must be $2-3$ orders of magnitude greater than in the molecular cloud, with $X_{\mathrm{H}_{2} \mathrm{CO}} \sim 3 \times 10^{-7}$ providing a mass consistent with the density lower-limit based mass. For a reasonable range of "core" masses, $20 M_{\odot}<M<110 M_{\odot}$, the implied abundance is $3 \times 10^{-7}>X_{\mathrm{H}_{2} \mathrm{CO}}>5 \times 10^{-8}$. The source e8 similarly requires high abundance to explain its emission. While these inferred abundances are high, they are consistent with measurements of $\mathrm{H}_{2} \mathrm{CO}$ abundance in the Orion Hot Core and Compact Ridge (Mangum et al. 1993). The enhanced abundance is likely caused by radiation-driven desorption of $\mathrm{H}_{2} \mathrm{CO}$ from dust grains (Shalabiea \& Greenberg 1994; van Dishoeck \& Blake 1998).

\subsection{The $d 4$ variable source and $\mathrm{H}_{2}$ region}

The source $\mathrm{d} 4 \mathrm{e}$ exhibits $\sim 20$-year timescale variability. $\mathrm{d} 4 \mathrm{e}$ has brightened from $<0.6 \mathrm{mJy}$ ( $3 \sigma$ upper limit) to $>1.0 \pm 0.06 \mathrm{mJy}$. We consider a few possibilities for the origin of this variable emission: a protostellar jet, a stellar wind, ionized accretion onto a star, or a knot in a high-velocity outflow. While we cannot

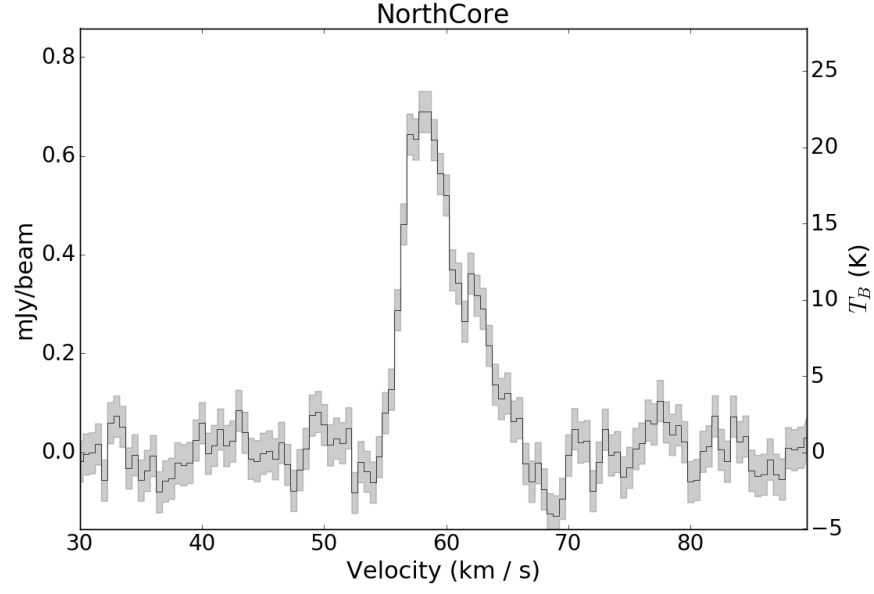

Fig. 13. Spectrum of the W51 North core in $\mathrm{o}-\mathrm{H}_{2} \mathrm{CO} 2_{1,1}-2_{1,2}$. The spectrum is the average over a $2^{\prime \prime} \times 1.5^{\prime \prime}$ elliptical aperture extracted from the Briggs-weighted cube (about $14 \times$ the beam area). The grey shading shows the $1 \sigma$ errorbars.

definitively rule out any of these scenarios, we favor the outflowISM interaction because it explains the coincidence of $\mathrm{d} 4 \mathrm{e}$ and $\mathrm{d} 4 \mathrm{w}$ with extended NIR $\mathrm{H}_{2}$ and [Fe II] and X-ray continuum emission.

Protostellar jets exhibit radio emission that can be variable. Radio variability has been observed in nearby star-forming regions (Liu et al. 2014; Forbrich \& Wolk 2013) and associated with variability in accretion or jet related processes, but all sources seen in these regions are orders of magnitude fainter than $\mathrm{d} 4 \mathrm{e}$, so a jet from a low-mass star is unlikely to be responsible for the emission. Radio jets from high-mass stars, e.g., the W75N jet (Carrasco-González et al. 2015), are also too faint: the expanding jet in $\mathrm{W} 75 \mathrm{~N}$ would have a flux density $S_{5 \mathrm{GHz}}<0.1 \mathrm{mJy}$ at the distance of W51. Nonetheless, jets are highly variable and may interact with a surrounding medium of dramatically different density in different regions, resulting in wildly different luminosities. We therefore cannot rule out the possibility that the emission comes from the formation of a jet, and it is even 


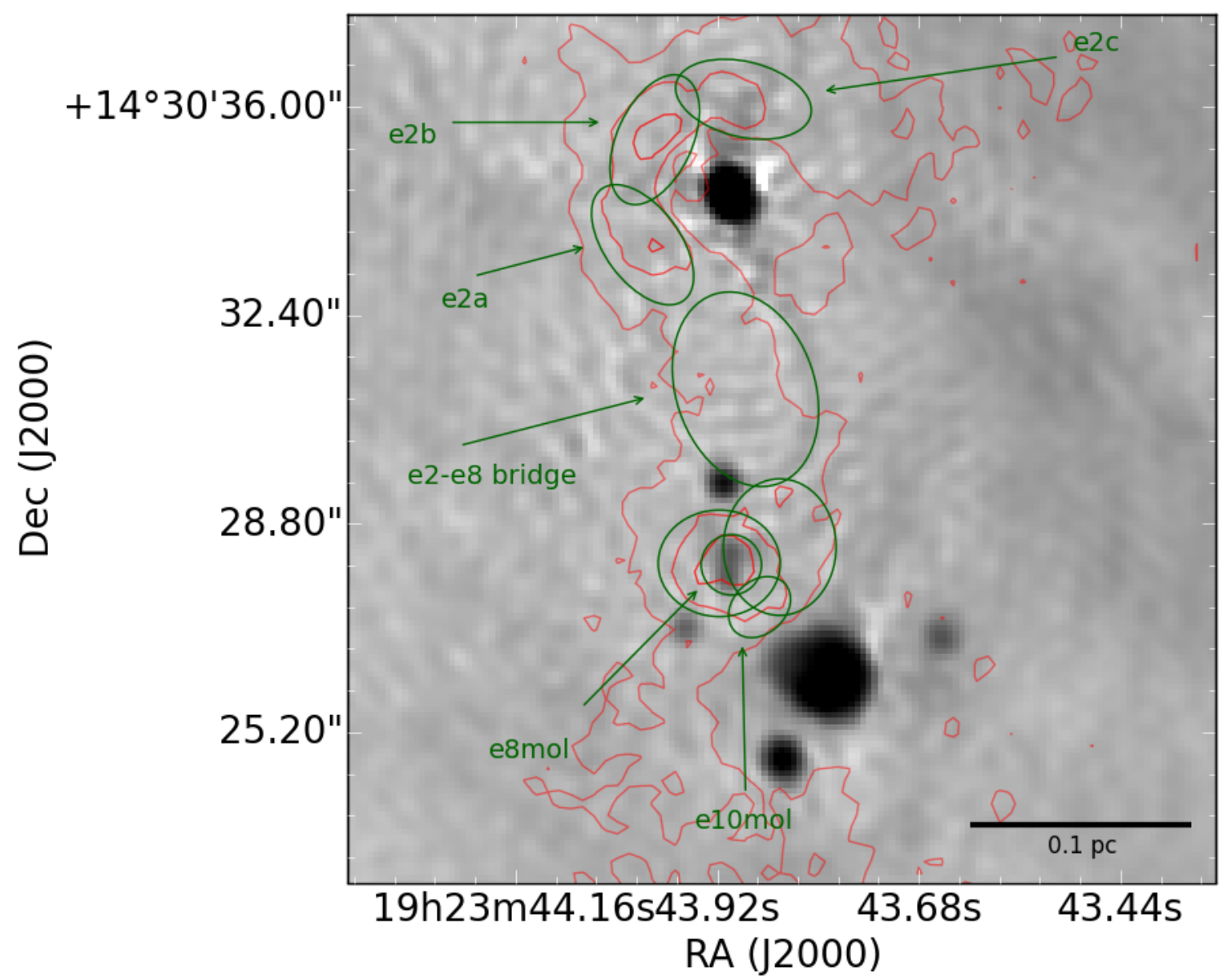

Fig. 14. Peak intensity contours (red) of the naturally weighted $\mathrm{H}_{2} \mathrm{CO} 2_{1,1}-2_{1,2}$ emission $\left(\theta_{\mathrm{FWHM}} \approx 1^{\prime \prime}\right)$ from 55 to $62 \mathrm{~km} \mathrm{~s} \mathrm{k}^{-1}$ in the e1/e 2 region superposed on the $\mathrm{Ku}$-band continuum image $\left(\theta_{\mathrm{FWHM}} \approx 0.33^{\prime \prime}\right)$. The contours are at 2, 4, $6 \mathrm{mJy} / \mathrm{beam}$. The green circles show the apertures used for the spectra shown in Figs. 16 and 17. The larger apertures around e8mol and e10mol are e8mol_ext and e10mol_ext. The feature denoted "e2-e8 bridge" is a diffuse feature that is seen in the naturally-weighted but not in the robust-weighted images. e2a and e $2 \mathrm{~b}$ in this figure are both associated with the SMA and VLA source e2e (Shi et al. 2010b; Goddi et al. 2016). e2c is associated with the source e2nw.

plausible that the $\mathrm{d} 4$ pair represents two opposing jets forming the base of a larger outflow structure.

In keeping with the SED interpretation from Sect. 4.2, it is possible that the $\mathrm{d} 4$ sources are massive stars with strong stellar winds. In this interpretation, the overlap with the NIR $\mathrm{H}_{2}$ and [Fe II] outflow emission (Fig. 18, Hodapp \& Davis 2002) may either be pure coincidence, or the winds of the massive stars may be interacting directly with the outflow from IRS2. While it is highly unlikely that an outflow would impact these rare windy stars, the interaction would provide a natural explanation for the large line widths observed in the near infrared and for the presence of radio emission. However, the weakness of the NIR $K$ band detection $\left(m_{K} \sim 15\right.$, and the emission is extended) suggests no massive stars are present.

The radio emission could come from ionized jets or magnetospheric emission associated with accreting young stars. However, the brightest $\mathrm{T}$ Tauri stars in the Orion Nebula Cluster (ONC) have $L_{\mathrm{rad}}<10^{28} \mathrm{erg} \mathrm{s}^{-1}$, or a flux density $S_{5 \mathrm{GHz}}<$ 0.1 mJy (Forbrich \& Wolk 2013; Zapata et al. 2004), so it is unlikely that these are accreting low- or intermediate- mass stars.

These sources could be very compact $\mathrm{HCH}$ II regions, in which case their variability may be caused by variable accretion flow (Peters et al. 2010a; Galván-Madrid et al. 2011; De Pree et al. 2014). In this scenario, the source d4e was fainter $20 \mathrm{yr}$ ago because the accretion rate was higher, which meant that the $\mathrm{HCH}$ II region was smaller.

The interaction between protostellar outflows and the ISM can result in radio and $\mathrm{X}$-ray emission at great distances from the driving source (e.g., HH80/81, López-Santiago et al. 2013; Masqué et al. 2015). There is a region of $\mathrm{H}_{2}$ emission, MHO 2419, coincident with d4 (Fig. 18, Hodapp \& Davis 2002; Froebrich et al. 2011). The $\mathrm{H}_{2}$ and [Fe II] emission come from bow shocks based on morphological and velocity structure in the Hodapp \& Davis (2002) spectra, and these bow shocks point back to an origin in IRS2. The high-velocity [Fe II] features $\left( \pm 150 \mathrm{~km} \mathrm{~s}^{-1}\right)$ are a strong indication that this is a bow shock from a high-velocity outflow. It is therefore possible that the $\mathrm{d} 4$ sources are emission features within the bow shock. The variability arises because the shocks are short-lived transient phenomena. The flux density of $\mathrm{d} 4 \mathrm{e}$ and $\mathrm{d} 4 \mathrm{w}$ is consistent with that observed in HH 80/81 (Masqué et al. 2015) to within an order of magnitude, though $\mathrm{d} 4$ is more luminous. Given all of the observed coincident features, $\mathrm{d} 4$ is best explained as an outflow knot.

\subsection{The velocity dispersion in the W51e cluster}

We detect $\mathrm{H} 77 \alpha$ toward five of the eight hyper/ultra compact $\mathrm{H}$ II regions in the W51e1/e2 cluster; four of these five are firm 


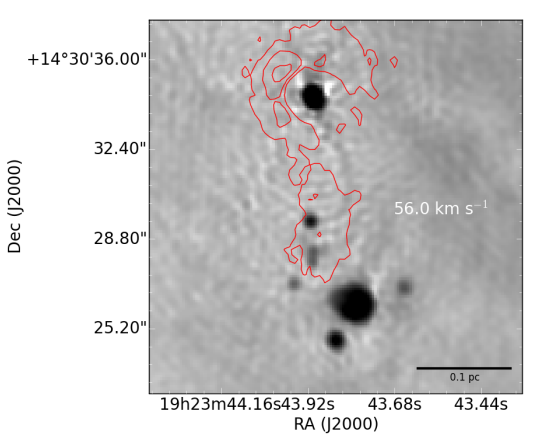

(a)

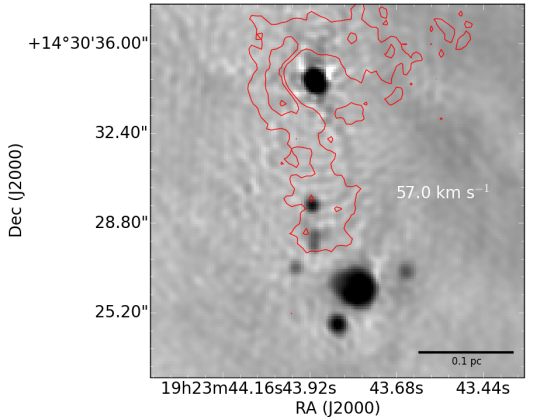

(b)

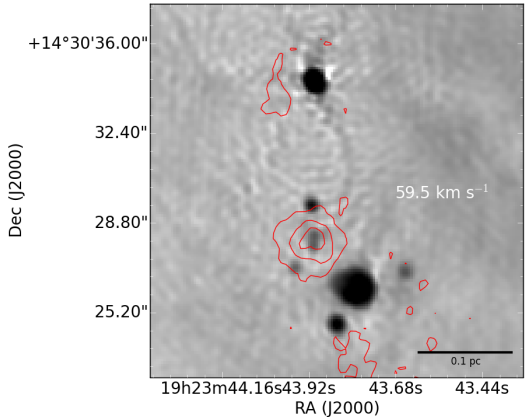

(c)

Fig. 15. Contours of the $\mathrm{H}_{2} \mathrm{CO} 2_{1,1}-2_{1,2}$ naturally weighted emission in the W51 Main region at 3 velocities superposed on the $\mathrm{Ku}$-band $14.5 \mathrm{GHz}$ continuum map. a) shows the peak velocity of the e 2 core, where the center of the core is missed because it is in absorption against the bright continuum peak; b) shows the overlap velocity; and c) shows the e8 core

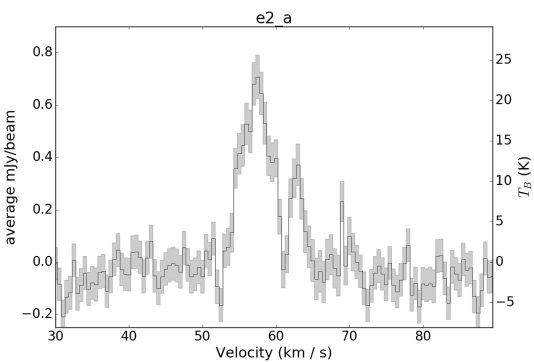

(a)

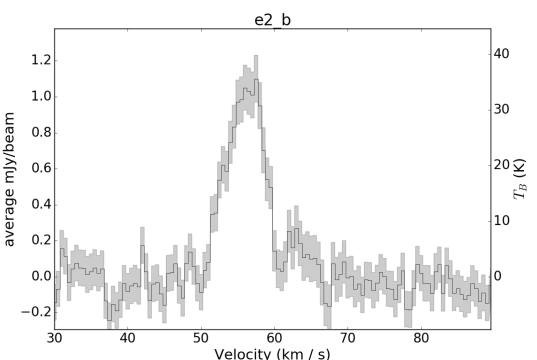

(b)

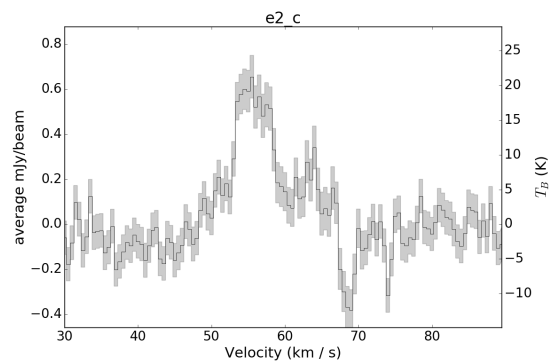

(c)

Fig. 16. Spectra of the $2_{1,1}-2_{1,2}$ emission around W51e 2 at three positions a) north/northwest b) northeast c) southeast of the e $2 \mathrm{HCH}$ II region. See Fig. 14 to see where these were extracted. The left axis shows the average brightness over the aperture in mJy/beam, while the right axis shows the brightness temperature.

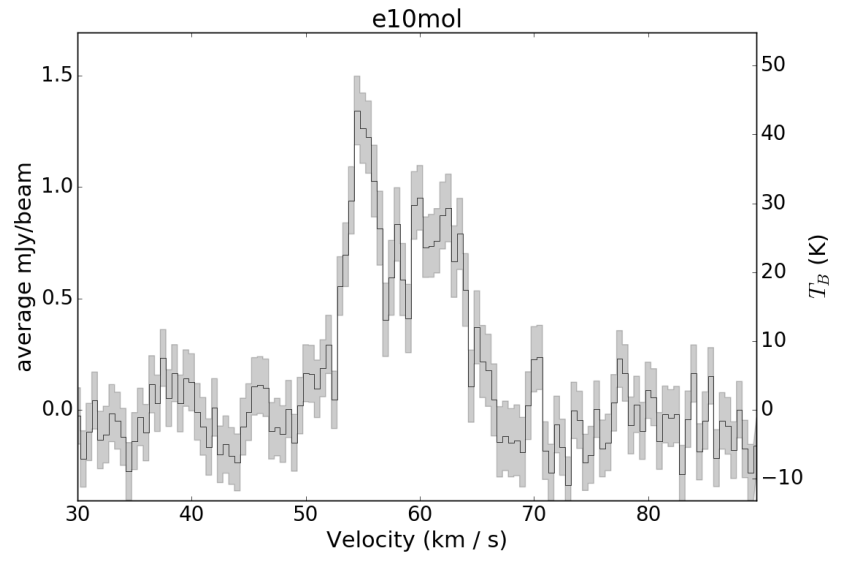

(a)

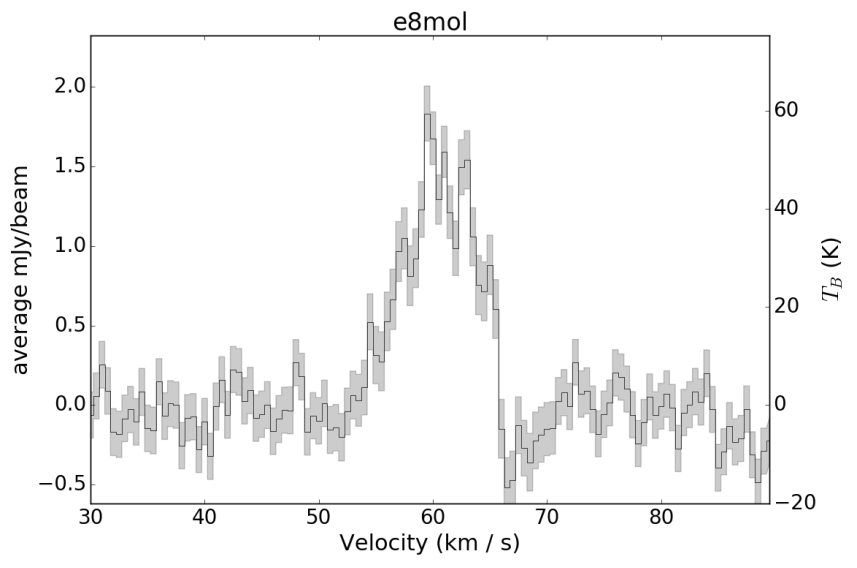

(b)

Fig. 17. Spectra of the $2_{1,1}-2_{1,2}$ emission around W51e1. See Fig. 14 to see where these were extracted. The left axis shows the average brightness over the aperture in $\mathrm{mJy} /$ beam, while the right axis shows the brightness temperature.

detections, one is weak. We also determine a velocity from the $\mathrm{H}_{2} \mathrm{CO}$ emission from e8mol and more uncertainly from e10mol. Although the molecular and ionized emission trace different processes, we treat the line-of-sight velocities of both tracers as though they are representative of the stellar velocity for this analysis. With such compact H II regions, the ionized gas is unlikely to be significantly shifted from the stellar velocity. The core emission is likely to be dominated by the densest component nearest the central accreting source.
The resulting 1D velocity dispersion is $\sigma=2.0 \mathrm{~km} \mathrm{~s}^{-1}$ if we exclude the uncertain sources e9 and e10mol; with e9 and e10mol included the dispersion increases to $3.6 \mathrm{~km} \mathrm{~s}^{-1}$. This velocity dispersion is confined to $r<5.4^{\prime \prime}$ or $r<0.13 \mathrm{pc}$, though the (proto)stars are not symmetrically distributed. The more compact and round e1 subcluster, which contains the symmetrically-distributed sources e1, e3, e4, e9, e8mol and e10mol, has a velocity dispersion $\sigma=2.0 \mathrm{~km} \mathrm{~s}^{-1}$ in $r=$ $2.9^{\prime \prime}(0.07 \mathrm{pc})$. 
If we assume all six sources in the e1 subcluster represent OB stars $\left(M>8 M_{\odot}\right)$ and the stellar masses are distributed according to a standard Kroupa (2001) mass function, the cluster should be star-dominated with a mass $M_{\text {cluster }}=600 \pm 170 M_{\odot}$. The stellar virial mass in the e 1 subcluster implied by its velocity dispersion and radius is $M_{\mathrm{vir}, *}=670 M_{\odot}$, given that (1) the stars are virialized, which is appropriate if the stellar mass is dominant (Kruijssen et al. 2012) and (2) the stars follow a Plummer (1911) profile (cf. Portegies Zwart et al. 2010, though the inferred mass is insensitive to the assumed radial profile). The agreement between the luminous and virial masses hints that the e 1 subcluster is stellar dominated, which can be checked independently by putting constraints on the gas mass. The lower limit on the gas density from detecting $\mathrm{H}_{2} \mathrm{CO}$ in emission is $n>10^{6} \mathrm{~cm}^{-3}$, which implies a gas mass $M_{\text {gas }}>100 M_{\odot}$ within the e1 subcluster. Overall, the observations of e1 are consistent with it being a stellar-dominated but nonetheless gas-rich $\left(M_{\mathrm{gas}} / M_{*}>0.15\right)$ system.

Given the mass of W51 Main and its $\sim 10 \mathrm{~km} \mathrm{~s}^{-1}$ escape velocity, all of these (proto)stars are bound to the overall cluster gas. In the innermost region, where the stellar mass is dominant, the stars are also likely to be bound to themselves.

\subsection{Constraints on the bulk outflow from IRS2}

In Sect. 3.4, we noted that the velocity structure of the RRL emission in W51 IRS2 is consistent with outflowing ionized gas. We use the RRL emission to constrain the ionized gas mass loss rate from this cluster. The outflowing ionized gas we observe is unbound from the system, moving at $\sim 25 \mathrm{~km} \mathrm{~s}^{-1}$ relative to the cluster rest velocity, which is greater than the escape velocity $v_{\text {esc }} \approx 8 \mathrm{~km} \mathrm{~s}^{-1}$ assuming $M_{\text {cluster }}=10^{4} M_{\odot}$ and $r_{\text {protocluster gas }}=1.5 \mathrm{pc}$. The remaining ionized gas is bound to the system, with thermal line width $c_{\mathrm{s}} \approx 6.7 \mathrm{~km} \mathrm{~s}^{-1}$ assuming $T_{\mathrm{e}}=7.5 \times 10^{4} \mathrm{~K}$ (Ginsburg et al. 2015).

We use Equation 14.28 of Wilson et al. (2009) to determine the emission measure:

$E M=5.2 \times 10^{-4}\left(\frac{T_{\mathrm{B}}}{\mathrm{K}}\right)\left(\frac{T_{\mathrm{e}}}{\mathrm{K}}\right)^{3 / 2}\left(\frac{\Delta v}{\mathrm{kHz}}\right) \mathrm{cm}^{-6} \mathrm{pc}$.

To estimate an upper limit on the outflow mass, we use the peak brightness in the shell at $37.5 \mathrm{~km} \mathrm{~s}^{-1}$ of $S_{v}=1.7 \mathrm{mJy}$ beam $^{-1}$ in a $10 \mathrm{~km} \mathrm{~s}^{-1}$ band $\left(T_{B}=60 \mathrm{~K}\right.$; see Fig. 5 , panel 4$)$ and assume it fills the entire shell with $r=0.25 \mathrm{pc}$. The resulting emission measure is $E M \approx 10^{7} \mathrm{~cm}^{-6} \mathrm{pc}$, which for a shell with width 0.03 pc yields a density $n_{\mathrm{e}} \approx 2.4 \times 10^{4} \mathrm{~cm}^{-3}$ and a mass $M_{\text {shell }}=3.5 M_{\odot}$. For a more conservative upper limit, we assume the entire surface area (a circle, rather than a shell) has the same emission measure, resulting in $M_{\text {outflow }}=12 M_{\odot}$. The shell has a velocity difference from the peak of the HII region emission $\Delta v=25 \mathrm{~km} \mathrm{~s}^{-1}$, which at its current radius translates to a time $t=r / \Delta v=7.5 \mathrm{kyr}$. The implied upper limit mass loss rate is $\dot{M}=1.6 \times 10^{3} M_{\odot} \mathrm{Myr}^{-1}$, which gives an evaporation timescale for this $M>10^{4} M_{\odot}$ clump $t>6 \mathrm{Myr}$. The free-fall time averaged over the full volume of the protocluster gas is $t_{\mathrm{ff}}<0.5 \mathrm{Myr}$ assuming $n=10^{4} \mathrm{~cm}^{-3}$ within $R=1.5 \mathrm{pc}$, so the photoevaporation timescale is at least an order of magnitude longer. However, the molecular gas is centrally condensed (most of the volume has no molecular gas) and under pressure from an HII region, so the local gas density is likely to be much higher and the collapse timescale therefore shorter. Given the conservative assumptions adopted above, the true photoevaporative mass loss is likely an order of magnitude lower, suggesting that this mass loss mechanism is negligible at present.

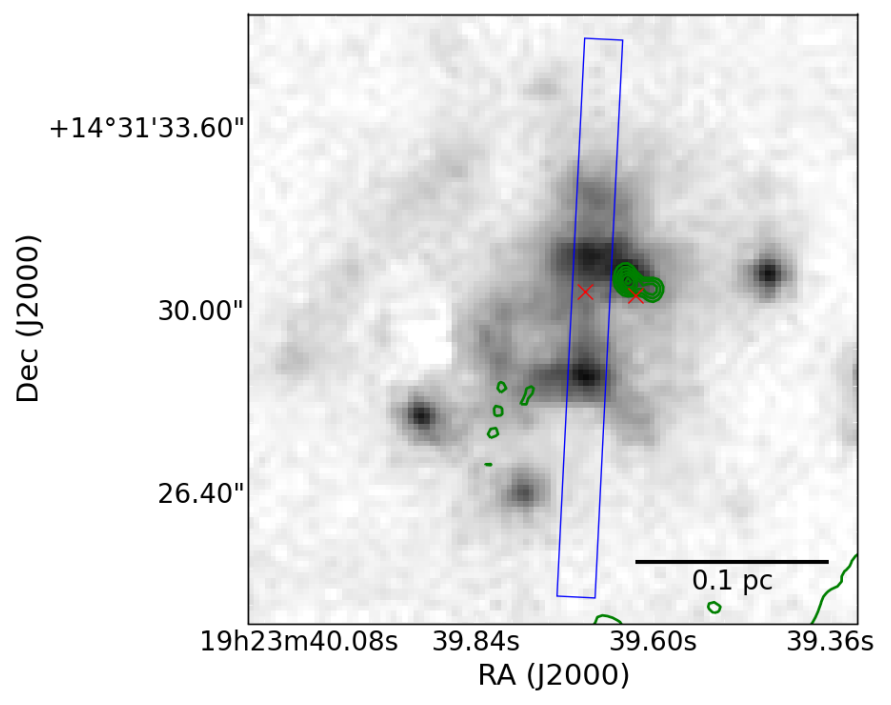

Fig. 18. A UWISH2 $\mathrm{H}_{2}$ image with $K u$-band contours of sources d4e and $\mathrm{d} 4 \mathrm{w}$ overlaid at $0.15,0.30,0.45,0.60,0.75$, and $0.90 \mathrm{mJy} / \mathrm{beam}$. The box shows the slit position used in Hodapp \& Davis (2002), in which [Fe II] emission spanning $>200 \mathrm{~km} \mathrm{~s}^{-1}$ was detected. The X's mark positions of MOXC X-ray sources (Townsley et al. 2014). Both radio sources are considered associated with the closer MOXC source in Table 8. This figure is discussed in Sect. 4.4.

\section{Discussion}

\subsection{The massive stars and the clusters}

There are three distinct massive star populations identified in W51: W51 IRS2, W51e1/e2, and the new W51e20 group. The W51 IRS2 cluster is closer to exhausting its available gas reservoir than the W51e1/e2 cluster, since it is visible in the nearinfrared. The presence of many stars with no ongoing accretion, along with the large volumes that are not occupied by dense gas, suggest the IRS2 cluster is older than W51e1/e2. The e1/e2 protocluster shows no signs of gas ejection, has no holes through which short-wavelength radiation is escaping, and contains no demonstrably non-accreting stars. Because they have no evident surrounding dense gas or suggestions of ongoing accretion, the W51e20 stars are likely the oldest in the W51A system.

However, W51e20 and the related W51e candidate CWBs may constitute an unclustered population. The Chandra MOXC catalog (Townsley et al. 2014) reveals that the most evident cluster is W51 IRS2, and while there is a mild overdensity associated with the W51 Main region, it is more amorphous than centrally concentrated (Fig. 20). While it is possible that the e20 group will merge with the e1/e 2 cluster, the later evolutionary stage of the e20 group (they are not embedded or accreting) suggests that they formed separately and in smaller groupings.

The W51d CWB candidates, by contrast, are each isolated, and they are distributed around W51 IRS2 (Figs. 6, 2). These stars could therefore be runaways from the IRS2 cluster. Source d5 is the furthest of these in projection, so with a distance of $0.9 \mathrm{pc}$, its ejection time is $t=0.08\left(v_{*} / 10 \mathrm{~km} \mathrm{~s}^{-1}\right)^{-1}$ Myr. The runaway scenario provides an alternate explanation for the observed SEDs (Appendix B): synchrotron emission from the wind-ISM bow shock might generate the detected emission. This scenario can be readily verified if high $\left(>10 \mathrm{~km} \mathrm{~s}^{-1}\right)$ line-of-sight velocities to the near-infrared-visible stars are measured. 


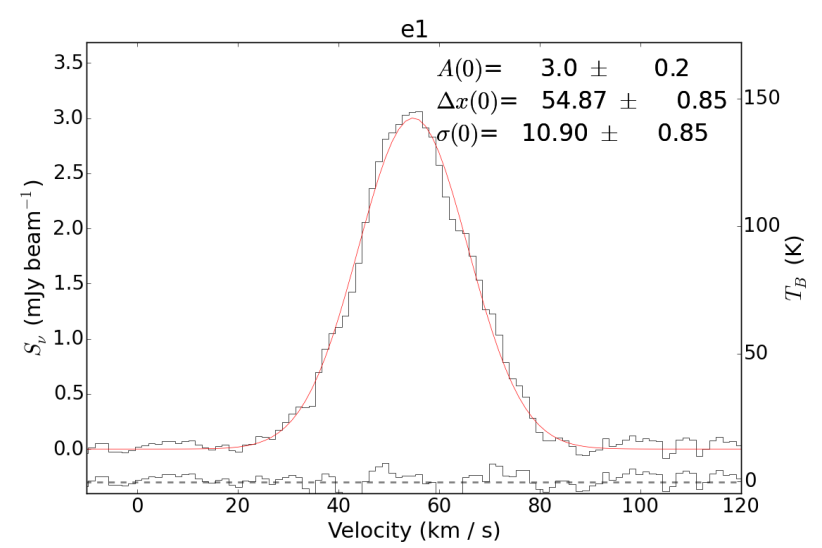

(a)

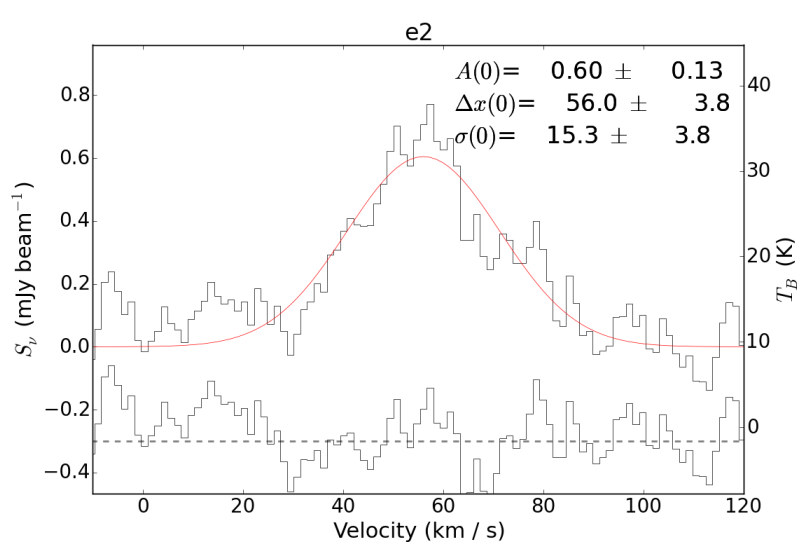

(b)

Fig. 19. H77 $\alpha$ spectra of W51e1 and W51e2. These velocities are used in conjunction with the $\mathrm{H}_{2} \mathrm{CO}$ emission fits to determine the velocity dispersion in Sect. 4.5. The red curve shows the fit. The lower spectrum shows the fit residual, with the dashed line indicating the zero level.

\subsection{The future evolution of the W51 clusters and molecular cloud}

The W51 clusters are capable of reaching a high star formation efficiency, since the escape velocity from them is greater than the sound speed in ionized gas (Matzner 2002; Ginsburg et al. 2012; Bressert et al. 2012; Dale et al. 2014) and the H II regions evidently leak optical photons, reducing the effectiveness of radiative and wind feedback. If they really are forming one or more young massive clusters, the gas within $\sim 1$ pc must be evacuated or exhausted to achieve a final appearance like that of NGC 3603 or Trumpler 14, our Galaxy's prototypical young massive clusters.

While the feedback from massive stars throughout W51 is intense, it is not halting star formation. There is evidence for ongoing star formation in both W51e1/e2 and IRS2 in the form of molecular cores (Sect. 4.3), ongoing accretion onto massive young stellar objects (MYSOs) like IRS2E (Figuerêdo et al. 2008), and the numerous HCH II regions that are likely to be accreting. In e1/e2, there is no sign that feedback has evacuated any gas yet, and the H II regions appear to be surrounded by molecular gas.

In IRS2, accretion is ongoing despite much of its volume being evacuated of molecular gas. Ionizing photons are escaping the inner cluster and ionized gas is being driven out of the inner cluster where the stars are forming. However, the timescale for complete gas evacuation in IRS2 at the presently observed mass loss rate is $>10 \times$ longer than the gas free-fall time on the $\sim 1$ parsec scale of the clump, and the star-forming gas is much more concentrated than this parsec-scale average (Sect. 4.6). Feedback is therefore progressing more slowly than star formation. The inefficiency of feedback in removing the star-forming gas supports the argument that the most massive clumps in the galaxy end their formation by exhausting their parent reservoir rather than by disrupting their parent cloud (Kruijssen et al. 2012; Matzner \& Jumper 2015).

The coexistence of distributed and clustered populations over a fairly compact region affects how their feedback couples to the local cloud. Because these populations are nearly coeval and spatially coincident, the feedback from the OB star population onto the gas within $\lesssim 5 \mathrm{pc}$ is not spherically symmetric as if it were injected from a single cluster. The distributed population will render the $\sim 5 \mathrm{pc}$-scale cloud more holey (porous) than would be implied by a smooth or even a very turbulent molecular cloud. These holes provide networks of low-density gaps through which radiation can escape, reducing the overall impact of radiative feedback on the cloud. Radiative escape helps explain why feedback has been inefficient at halting further star formation. We conclude that feedback only affects the low-density gas, while the high-density, star-forming gas is largely unaffected.

\subsubsection{Merging and age spread}

Given the high binding energy of the clusters and the proximity of many other stars, it is likely that some of the observed subclusters will merge. Merger or collapse of clusters is a requirement for the formation of massive clusters in the Galactic center and disk (Walker et al. 2015, 2016), so it is reasonable to expect similar dynamic evolution here as well.

If any of the observed subclusters merge (e.g., e1/e2 with e20-e22 or with IRS2), the resulting cluster will have an age spread greater than the present age spread. If we assume the e20 cluster is responsible for driving the nearest part of the W51 Main shell (W51 IRS1) at a separation of 0.35 parsecs with a Dtype ionization front at $v \sim 2 \mathrm{~km} \mathrm{~s}^{-1}$, we derive a lower limit on its age $t_{\mathrm{e} 20}>0.17 \mathrm{Myr}$. This lower limit is comparable to the gas free fall time in the cluster and is still consistent with the most accurate stellar age spread estimates available from photometry of young clusters (e.g., Kudryavtseva et al. 2012).

If instead e20-e22 are part of a distributed population that will not merge with e1/e2, the W51 protoclusters are embedded within an $\mathrm{OB}$ association. The $\mathrm{OB}$ association will dissolve over a few Myr while the clusters will survive. However, before this happens, the $\mathrm{OB}$ association is likely to shut off accretion onto the protoclusters. The radiative feedback from the OB association can readily ionize the lower-density molecular gas in the outskirts of the clusters. In this way, the cluster's final mass would be externally feedback regulated rather than, or perhaps in addition to, being self-regulated.

\subsubsection{Final mass}

The present stellar mass as inferred from the infrared luminosity (Sect. 4.1) is a lower limit on the final mass of the clusters, 


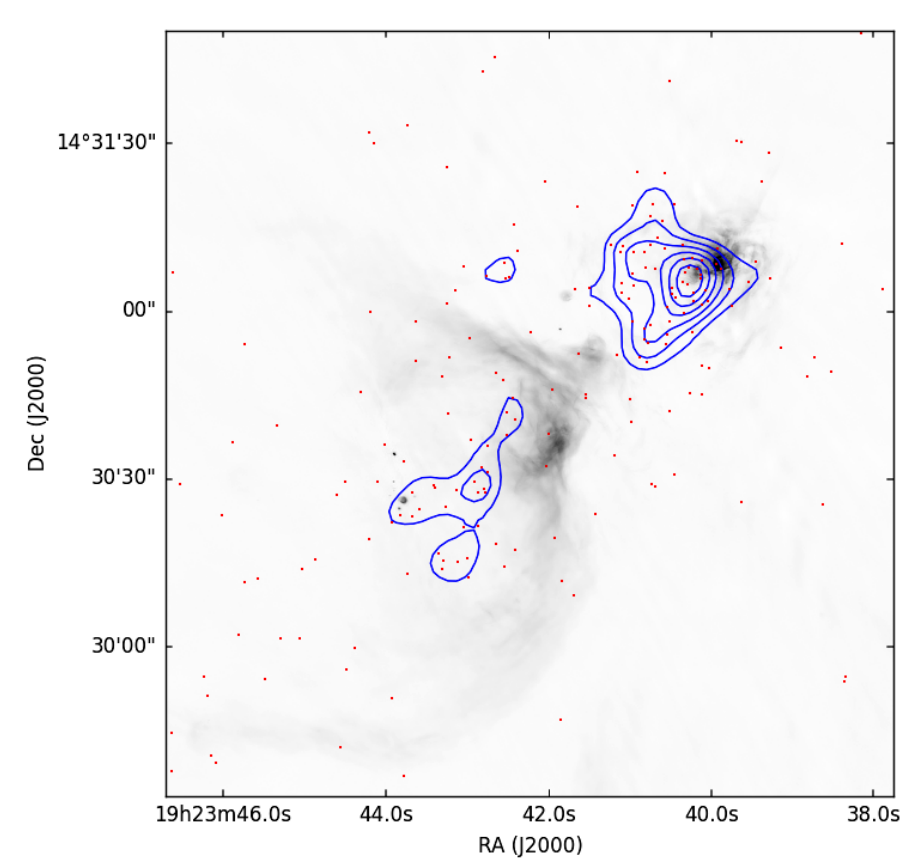

Fig. 20. Contours and individual points showing the X-ray source density from the MOXC (Townsley et al. 2014) catalog overlaid on the C-band image of W51. See Sect. 5.1.

i.e., the mass after the remaining gas has either been converted to stars or ejected from the system. The final mass of the combined clusters will be at least $\gtrsim 7 \times 10^{3}+5 \times 10^{4} \eta M_{\odot}$, where $5 \times 10^{4} M_{\odot}$ is the approximate dense gas mass within $\sim 2.5 \mathrm{pc}$ and $\eta$ is a star formation efficiency, likely to be at least $10 \%$ in the dense gas, so the stellar $M_{\text {final }}>1.2 \times 10^{4} M_{\odot}$. This is a conservative lower limit, as it may miss a substantial population of stars whose luminosity is not reprocessed into the infrared and it uses conservatively low estimates for the gas mass and the star formation efficiency.

\subsubsection{Cloud-scale feedback}

Since the free-fall time is short within these clusters $\left(t_{\mathrm{ff}}<\right.$ $0.5 \mathrm{Myr}$ out to $2.5 \mathrm{pc}$ containing $5 \times 10^{4} M_{\odot}$ of gas, i.e., the region containing both clusters), supernovae are not likely to affect their formation. However, once the clusters have exhausted gas locally, they will remain in the cloud long enough for the stars to evolve and die. Exhaustion and porosity also provide a relatively free line-of-sight from the clusters to the rest of the cloud. The binding energy of a $10^{6} M_{\odot}, r=50 \mathrm{pc}$ molecular cloud is only about $10^{51} \mathrm{erg}$, so a single supernova perfectly coupled to the gas could unbind it all (though typical energy delivered to molecular clouds is lower, $\sim 0.01-0.25$; see references in Kruijssen 2012, Appendix B). The 20 O-stars presently formed, plus at least that many expected to form, could provide enough energy to unbind the whole W51 GMC in a few Myr. However, the location of the clusters on the outskirts of the GMC imply a substantially lower coupling factor, suggesting instead that supernovae will only partially disrupt the cloud.

\subsubsection{Evidence against triggering}

The star formation ongoing within this cloud shows no signs of having been triggered by expanding $\mathrm{H}$ II regions, though there are multiple generations separated by temporal and spatial scales that might otherwise suggest a typical expanding-H II-regiondriven triggering scenario. The main regions of ongoing star formation at present are W51e1/e2 and IRS2. However, the most evident region of feedback is the W51 Main Shell, especially W51 IRS1 (Fig. A.2). Throughout the W51 Main Shell, there is no sign of any excess of newly formed protostars (Fig. 20) and there is a clear deficit of molecular gas in W51 IRS1 as traced by $\mathrm{C}^{18} \mathrm{O}$ (Fig. 11; Parsons et al. 2012). Where there is the strongest evidence of interacting and colliding H II region shells (Fig. 5 as discussed in Sect. 3.4), no sign of ongoing star formation is observed.

\section{Conclusions}

We present deep, high-resolution radio continuum images in many low-frequency bands and $\mathrm{H} 77 \alpha$ and $\mathrm{o}-\mathrm{H}_{2} \mathrm{CO} 2_{1,1}-2_{1,2}$ spectral line cubes from the Jansky Very Large Array with angular resolution $0.3^{\prime \prime}$ to $0.5^{\prime \prime}$. These observations have revealed many intriguing features of the W51 protocluster that together suggest that thermal and radiative feedback from massive stars is ineffective at halting star formation in massive protoclusters, similar to what has been concluded in other high-mass protoclusters (e.g. Ginsburg et al. 2012; Galvan-Madrid et al. 2013). These observations support theoretical models that require ineffective feedback as a necessary step in the formation of gravitationally bound stellar clusters.

Using the $\mathrm{H} 77 \alpha$ emission and $\mathrm{H}_{2} \mathrm{CO}$ emission and absorption line information, we have measured the velocity dispersion of the W51 e1 protocluster $\sigma_{v}=2.0 \mathrm{~km} \mathrm{~s}^{-1}$. Although this cluster consists of hypercompact and ultracompact H II regions, indicating that it is extremely gas-rich, the velocity dispersion implies a stellar virial mass that is consistent with an extrapolated IMF stellar mass, suggesting that the innermost cluster $(r<0.07 \mathrm{pc})$ is already dynamically star-dominated.

The SEDs of the point sources detected throughout the W51A region have peculiar shapes. Most of them exhibit an excess at low frequencies $(<5 \mathrm{GHz})$ that renders their overall SED shape inconsistent with either an optically thick or thin free-free spectrum. These sources are likely to be colliding wind binaries, exhibiting a combination of free-free and synchrotron emission.

We detected radio recombination line $(\mathrm{H} 77 \alpha)$ emission throughout W51. The RRLs trace a number of overlapping bubbles toward the W51 IRS1 emission peak and show that the IRS2 region has excavated a cavity along our line of sight.

The Lacy et al. (2007) high-velocity ionized jet is detected in $\mathrm{H} 77 \alpha$ at velocities consistent with the [Ne II] observations. The jet remains mysterious as there is still no direct information about what drives it.

We have detected the three massive cores from Zhang \& Ho (1997) in $\mathrm{H}_{2} \mathrm{CO} 2_{1,1}-2_{1,2}$ emission. For W51 North, we evaluated the mass and derived a lower limit $M>15 M_{\odot}$, though it is likely to be significantly higher. The $\mathrm{H}_{2} \mathrm{CO}$ abundance in these cores is enhanced compared to the surrounding molecular cloud by at least $100 \times$, with $X_{\mathrm{H}_{2} \mathrm{CO}} \sim 10^{-7}$, similar to the Orion BN/KL hot core (Mangum et al. 1993). In the W51e8 molecular core, we have found a compact continuum source, indicating unambiguously that these cores are forming massive stars.

Because of its association with high-mass protostellar cores, the $\mathrm{H}_{2} \mathrm{CO} 2_{1,1}-2_{1,2} 14.488 \mathrm{GHz}$ transition is a good tracer of early-stage massive star formation. Formaldehyde is apparently enhanced in abundance by 2-3 orders of magnitude over ISM values in high-mass protostellar cores, allowing it to be detected in emission at long wavelengths. This transition will be a powerful tool for studying the earliest stagest of high-mass 
star formation throughout the Galaxy with the Next Generation VLA or a Square Kilometer Array. Ongoing JVLA surveys (e.g., KuGARS, PI Thompson, http://library.nrao.edu/ proposals/catalog/10267) may detect a significant additional population of proto-O-stars.

The W51e1/e2 and IRS2 protoclusters appear to be highmass clusters forming within a larger OB association. The coexistence of the distributed and clustered OB-star populations suggests that external feedback may be partly responsible for halting protocluster feeding.

The weak outflow from IRS2 and ongoing accretion in IRS2 and e1/e2 suggest that gas exhaustion is a more important mechanism than expulsion in halting cluster growth. Recent theoretical models predict gas exhaustion to be a necessary step in the formation of gravitationally bound stellar clusters (e.g., Kruijssen 2012) and our results provide evidence of this process in action.

These results show that even the low-pressure interstellar medium of the Milky Way can reach densities high enough for gravitational collapse to form clusters on $<$ Myr timescales, rendering stellar feedback ineffective. Future observational studies aiming to understand the formation of massive stellar clusters should target regions with properties similar to W51, where gas exhaustion may be prevalent and the conditions therefore enable gravitationally bound clusters to form.

Acknowledgements. The National Radio Astronomy Observatory is a facility of the National Science Foundation operated under cooperative agreement by Associated Universities, Inc. J.M.D.K. gratefully acknowledges financial support in the form of a Gliese Fellowship and an Emmy Noether Research Group from the Deutsche Forschungsgemeinschaft (DFG), grant number KR4801/1-1. This research made use of: APLpy, an open-source plotting package for Python hosted at http://aplpy.github.com, Astropy, a community-developed core Python package for Astronomy (Astropy Collaboration et al. 2013), ds9, a too for data visualization supported by the Chandra X-ray Science Center (CXC) and the High Energy Astrophysics Science Archive Center (HEASARC) with support from the JWST Mission office at the Space Telescope Science Institute for 3D visualization, myRadex, an alternative implementation of RADEX (http: //home.strw. leidenuniv.nl/ moldata/radex.html), pvextractor, a tool to extract position-velocity diagrams along arbitrary directions from data cubes, pyradex, a python wrapper of RADEX (http://home.strw.leidenuniv $\mathrm{nl} /$ moldata/radex.html), pyspeckit, an open-source spectral analysis and plotting package for Python hosted at http://pyspeckit.bitbucket.org (Ginsburg \& Mirocha 2011), spectralcube, a library for astronomical spectral data cubes, and wcsaxes, an open-source plotting library for Python hosted at https://wcsaxes.readthedocs.io/en/latest/.

\section{References}

Adamo, A., Kruijssen, J. M. D., Bastian, N., Silva-Villa, E., \& Ryon, J. 2015 MNRAS, 452, 246

Araya, E., Hofner, P., Goss, W. M., et al. 2007, ApJS, 170, 152

Astropy Collaboration, Robitaille, T., Tollerud, E. J., et al. 2013, A\&A, 558, A33

Barbosa, C. L., Blum, R. D., Conti, P. S., Damineli, A., \& Figuerêdo, E. 2008, ApJ, 678, L55

Bastien, P., Batrla, W., Henkel, C., et al. 1985, A\&A, 146, 86

Batrla, W., Wilson, T. L., \& Martin-Pintado, J. 1983, A\&A, 119, 139

Benjamin, R. A., Churchwell, E., Babler, B. L., et al. 2003, PASP, 115, 953

Bieging, J. H., Abbott, D. C., \& Churchwell, E. B. 1989, ApJ, 340, 518

Bressert, E., Ginsburg, A., Bally, J., et al. 2012, ApJ, 758, L28

Carrasco-González, C., Torrelles, J. M., Cantó, J., et al. 2015, Science, 348, 114

Condon, J. J., \& Ransom, S. 2007, Essential Radio Astronomy (Princeton University Press)

Dale, J. E., Ngoumou, J., Ercolano, B., \& Bonnell, I. A. 2014, MNRAS, 442, 694

De Becker, M., \& Raucq, F. 2013, A\&A, 558, A28

De Pree, C. G., Peters, T., Mac Low, M.-M., et al. 2014, ApJ, 781, L36

Evans, II, N. J., Sato, T., Morris, G., \& Zuckerman, B. 1975, ApJ, 199, 383
Figuerêdo, E., Blum, R. D., Damineli, A., Conti, P. S., \& Barbosa, C. L. 2008, AJ, 136, 221

Forbrich, J., \& Wolk, S. J. 2013, A\&A, 551, A56

Froebrich, D., Davis, C. J., Ioannidis, G., et al. 2011, MNRAS, 413, 480

Galván-Madrid, R., Keto, E., Zhang, Q., et al. 2009, ApJ, 706, 1036

Galvan-Madrid, R., Liu, H. B., Zhang, Z.-Y., et al. 2013, ApJ, 779, 121

Galván-Madrid, R., Peters, T., Keto, E. R., et al. 2011, MNRAS, 416, 1033

Ginsburg, A., \& Mirocha, J. 2011, PySpecKit: Python Spectroscopic Toolkit (Astrophysics Source Code Library)

Ginsburg, A., Darling, J., Battersby, C., Zeiger, B., \& Bally, J. 2011, ApJ, 736, 149

Ginsburg, A., Bressert, E., Bally, J., \& Battersby, C. 2012, ApJ, 758, L29

Ginsburg, A., Bally, J., Battersby, C., et al. 2015, A\&A, 573, A106

Goddard, Q. E., Bastian, N., \& Kennicutt, R. C. 2010, MNRAS, 405, 857

Goddi, C., Henkel, C., Zhang, Q., Zapata, L., \& Wilson, T. L. 2015, A\&A, 573, A109

Goddi, C., Ginsburg, A., \& Zhang, Q. 2016, 589, 44

Goldader, J. D., \& Wynn-Williams, C. G. 1994, ApJ, 433, 164

Harvey, P. M., Joy, M., Lester, D. F., \& Wilking, B. A. 1986, ApJ, 300, 737

Hodapp, K. W., \& Davis, C. J. 2002, ApJ, 575, 291

Johnston, K. J., Palmer, P., Wilson, T. L., \& Bieging, J. H. 1983, ApJ, 271, L89

Johnston, K. J., Henkel, C., \& Wilson, T. L. 1984, ApJ, 285, L85

Keto, E. 2003, ApJ, 599, 1196

Keto, E., \& Klaassen, P. 2008, ApJ, 678, L109

Keto, E., Zhang, Q., \& Kurtz, S. 2008, ApJ, 672, 423

Kroupa, P. 2001, MNRAS, 322, 231

Kruijssen, J. M. D. 2012, MNRAS, 426, 3008

Kruijssen, J. M. D., Maschberger, T., Moeckel, N., et al. 2012, MNRAS, 419, 841

Kudryavtseva, N., Brandner, W., Gennaro, M., et al. 2012, ApJ, 750, L44

Kumar, M. S. N., Kamath, U. S., \& Davis, C. J. 2004, MNRAS, 353, 1025

Kurtz, S. 2002, in Hot Star Workshop III: The Earliest Phases of Massive Star Birth, ed. P. Crowther, ASP Conf. Ser., 267, 81

Kutner, M. L., Evans, II, N. J., \& Tucker, K. D. 1976, ApJ, 209, 452

Lacy, J. H., Jaffe, D. T., Zhu, Q., et al. 2007, ApJ, 658, L45

Liu, H. B., Galván-Madrid, R., Forbrich, J., et al. 2014, ApJ, 780, 155

Longmore, S. N., Kruijssen, J. M. D., Bastian, N., et al. 2014, Protostars and Planets VI, 291

López-Santiago, J., Peri, C. S., Bonito, R., et al. 2013, ApJ, 776, L22

Loren, R. B., Wootten, A., Sandqvist, A., \& Bernes, C. 1980, ApJ, 240, L165

Loren, R. B., Sandqvist, A., \& Wootten, A. 1983, ApJ, 270, 620

Lucas, P. W., Hoare, M. G., Longmore, A., et al. 2008, MNRAS, 391, 136

Madau, P., \& Dickinson, M. 2014, ARA\&A, 52, 415

Mangum, J. G., \& Shirley, Y. L. 2015, PASP, 127, 266

Mangum, J. G., \& Wootten, A. 1993, ApJS, 89, 123

Mangum, J. G., Wootten, A., \& Plambeck, R. L. 1993, ApJ, 409, 282

Mangum, J. G., Darling, J., Menten, K. M., \& Henkel, C. 2008, ApJ, 673, 832

Martin-Pintado, J., Wilson, T. L., Gardner, F. F., \& Henkel, C. 1983, A\&A, 117 145

Masqué, J. M., Rodríguez, L. F., Araudo, A., et al. 2015

Matzner, C. D. 2002, ApJ, 566, 302

Matzner, C. D., \& Jumper, P. H. 2015, ApJ, 815, 68

McMullin, J. P., Waters, B., Schiebel, D., Young, W., \& Golap, K. 2007, in Astronomical Data Analysis Software and Systems XVI, eds. R. A. Shaw, F. Hill, \& D. J. Bell, ASP Conf. Ser., 376, 127

Mehringer, D. M. 1994, ApJS, 91, 713

Molinari, S., et al. 2016, ArXiv e-prints

Molinari, S., Swinyard, B., Bally, J., et al. 2010, A\&A, 518, L100

Moscadelli, L., Cesaroni, R., Sánchez-Monge, Á., et al. 2013, A\&A, 558, A145

Moscadelli, L., Sánchez-Monge, Á., Goddi, C., et al. 2016, A\&A, 585, A71

Okumura, S.-I., Mori, A., Nishihara, E., Watanabe, E., \& Yamashita, T. 2000, ApJ, 543, 799

Panagia, N., \& Felli, M. 1975, A\&A, 39, 1

Parsons, H., Thompson, M. A., Clark, J. S., \& Chrysostomou, A. 2012, MNRAS, 424, 1658

Pecaut, M. J., \& Mamajek, E. E. 2013, ApJS, 208, 9

Peters, T., Banerjee, R., Klessen, R. S., et al. 2010a, ApJ, 711, 1017

Peters, T., Mac Low, M.-M., Banerjee, R., Klessen, R. S., \& Dullemond, C. P. 2010b, ApJ, 719, 831

Plummer, H. C. 1911, MNRAS, 71, 460

Portegies Zwart, S. F., McMillan, S. L. W., \& Gieles, M. 2010, ARA\&A, 48, 431

Reynolds, S. P. 1986, ApJ, 304, 713

Sato, M., Reid, M. J., Brunthaler, A., \& Menten, K. M. 2010, ApJ, 720, 1055

Shalabiea, O. M., \& Greenberg, J. M. 1994, A\&A, 290, 266

Shi, H., Zhao, J.-H., \& Han, J. L. 2010a, ApJ, 718, L181

Shi, H., Zhao, J.-H., \& Han, J. L. 2010b, ApJ, 710, 843 
Sievers, A. W., Mezger, P. G., Bordeon, M. A., et al. 1991, A\&A, 251, 231

Surcis, G., Vlemmings, W. H. T., van Langevelde, H. J., \& Hutawarakorn Kramer, B. 2012, A\&A, 541, A47

Tanaka, K. E. I., Tan, J. C., \& Zhang, Y. 2016, ApJ, 818, 52

Townsley, L. K., Broos, P. S., Garmire, G. P., et al. 2014, ApJS, 213, 1

Traficante, A., Calzoletti, L., Veneziani, M., et al. 2011, MNRAS, 416, 2932

Urquhart, J. S., Moore, T. J. T., Csengeri, T., et al. 2013, MNRAS, 435, 400

Urquhart, J. S., Figura, C. C., Moore, T. J. T., et al. 2014a, MNRAS, 437, 1791

Urquhart, J. S., Moore, T. J. T., Csengeri, T., et al. 2014b, MNRAS, 443, 1555

Vacca, W. D., Garmany, C. D., \& Shull, J. M. 1996, ApJ, 460, 914

van Dishoeck, E. F., \& Blake, G. A. 1998, ARA\&A, 36, 317

Wadiak, E. J., Wilson, T. L., Rood, R. T., \& Johnston, K. J. 1985, ApJ, 295, L43

Walker, D. L., Longmore, S. N., Bastian, N., et al. 2015, MNRAS, 449, 715

Walker, D. L., Longmore, S. N., Bastian, N., et al. 2016, MNRAS, 457, 4536

Wilson, T. L., \& Johnston, K. J. 1989, ApJ, 340, 894
Wilson, T. L., Martin-Pintado, J., Gardner, F. F., \& Henkel, C. 1982, A\&A, 107, L10

Wilson, T. L., Rohlfs, K., \& Hüttemeister, S. 2009, Tools of Radio Astronomy (Springer-Verlag)

Wright, A. E., \& Barlow, M. J. 1975, MNRAS, 170, 41

Xu, Y., Reid, M. J., Menten, K. M., et al. 2009, ApJ, 693, 413

Yusef-Zadeh, F. 1990, ApJ, 361, L19

Zapata, L. A., Rodríguez, L. F., Kurtz, S. E., O’Dell, C. R., \& Ho, P. T. P. 2004 ApJ, 610, L121

Zapata, L. A., Palau, A., Ho, P. T. P., et al. 2008, A\&A, 479, L25

Zapata, L. A., Ho, P. T. P., Schilke, P., et al. 2009, ApJ, 698, 1422

Zapata, L. A., Tang, Y.-W., \& Leurini, S. 2010, ApJ, 725, 1091

Zeiger, B., \& Darling, J. 2010, ApJ, 709, 386

Zhang, Q., \& Ho, P. T. P. 1995, ApJ, 450, L63

Zhang, Q., \& Ho, P. T. P. 1997, ApJ, 488, 241 


\section{Appendix A: Figures labeling point sources and diffuse regions}

We include additional versions of Fig. A.1 with labels for the compact (Fig. 2) and extended (Fig. A.2) H II regions.

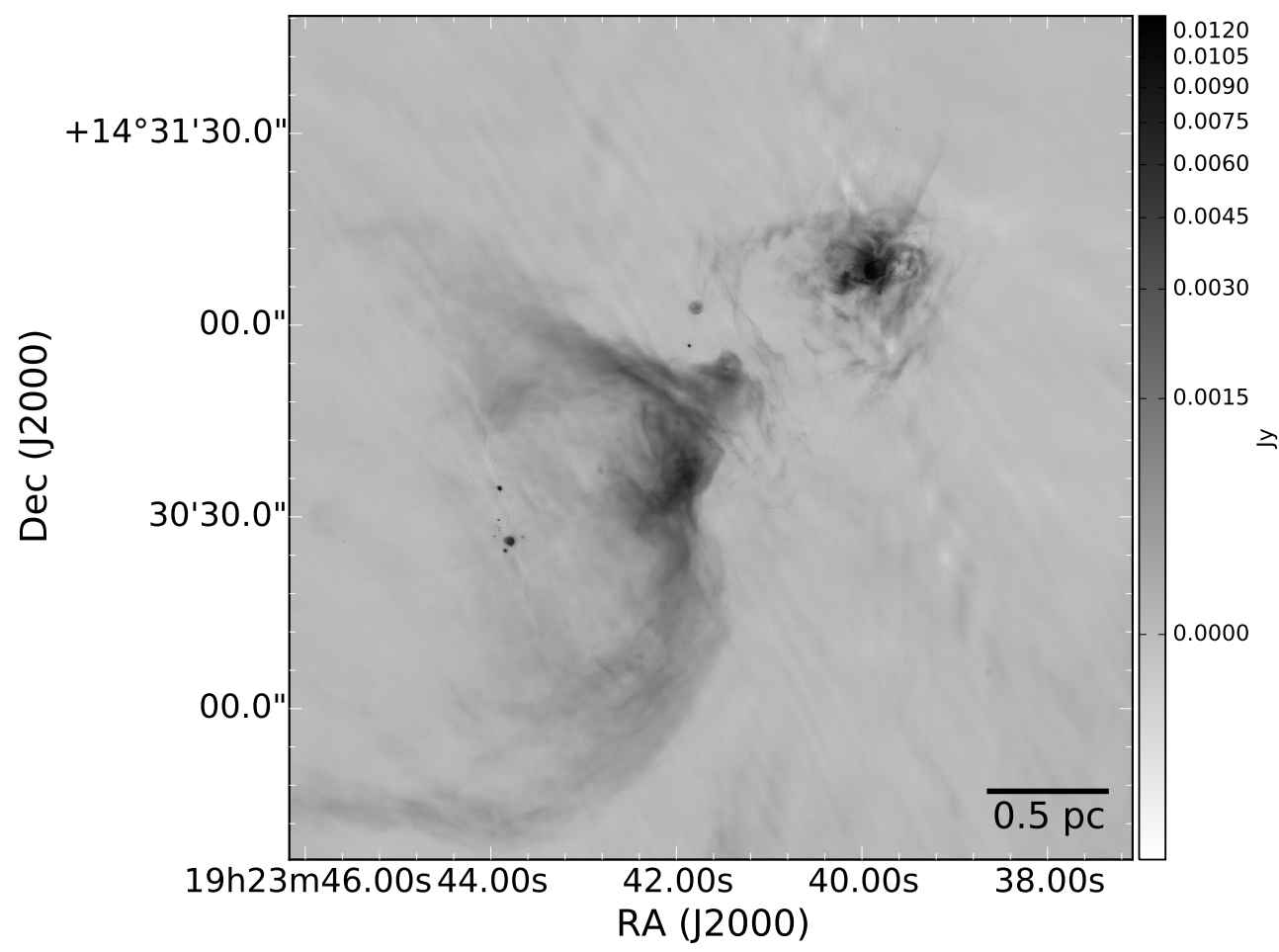

Fig. A.1. $C$-band image of the W51 region produced with a combination of JVLA C and A array data using uniform weighting. Annotated versions of this figure identifying the regions discussed are available in the Appendix.

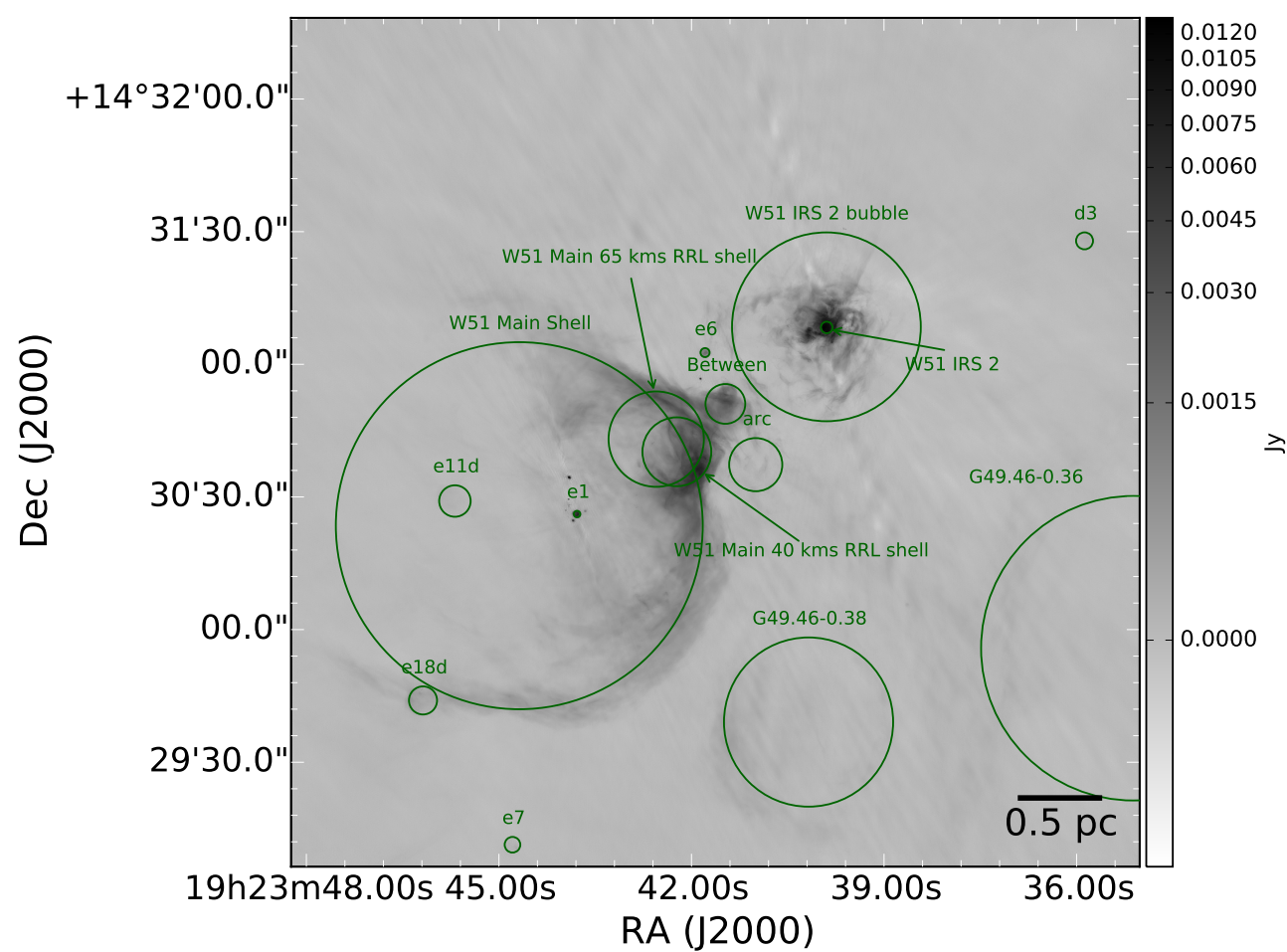

Fig. A.2. $C$-band image of the W51A region with diffuse $\mathrm{H}$ II regions labeled. The labels are above the circles except where arrows are used. The regions labeled W51 Main 65 kms RRL shell and W51 Main 40 kms RRL shell are part of W51 IRS1 (e.g., Zhang \& Ho 1997); we have given them independent labels here because we are now able to kinematically distinguish them in RRL emission and because they are evidently part of (or interacting with) a large shell centered near W51 Main. 


\section{Appendix B: Point source photometry catalog}

The full version of the point source photometry catalog is available in digital form from https://github.com/keflavich/ paper_w51_evla/blob/master/tables/EVLA_VLA_PointSourcePhotometry.ipac. A sample is shown in Table 4 and the full table is available at the CDS (Table A.1).

Figure B.1 shows cutout images with apertures for the source d4e. Additional figures for all candidates are included in a supplemental document.
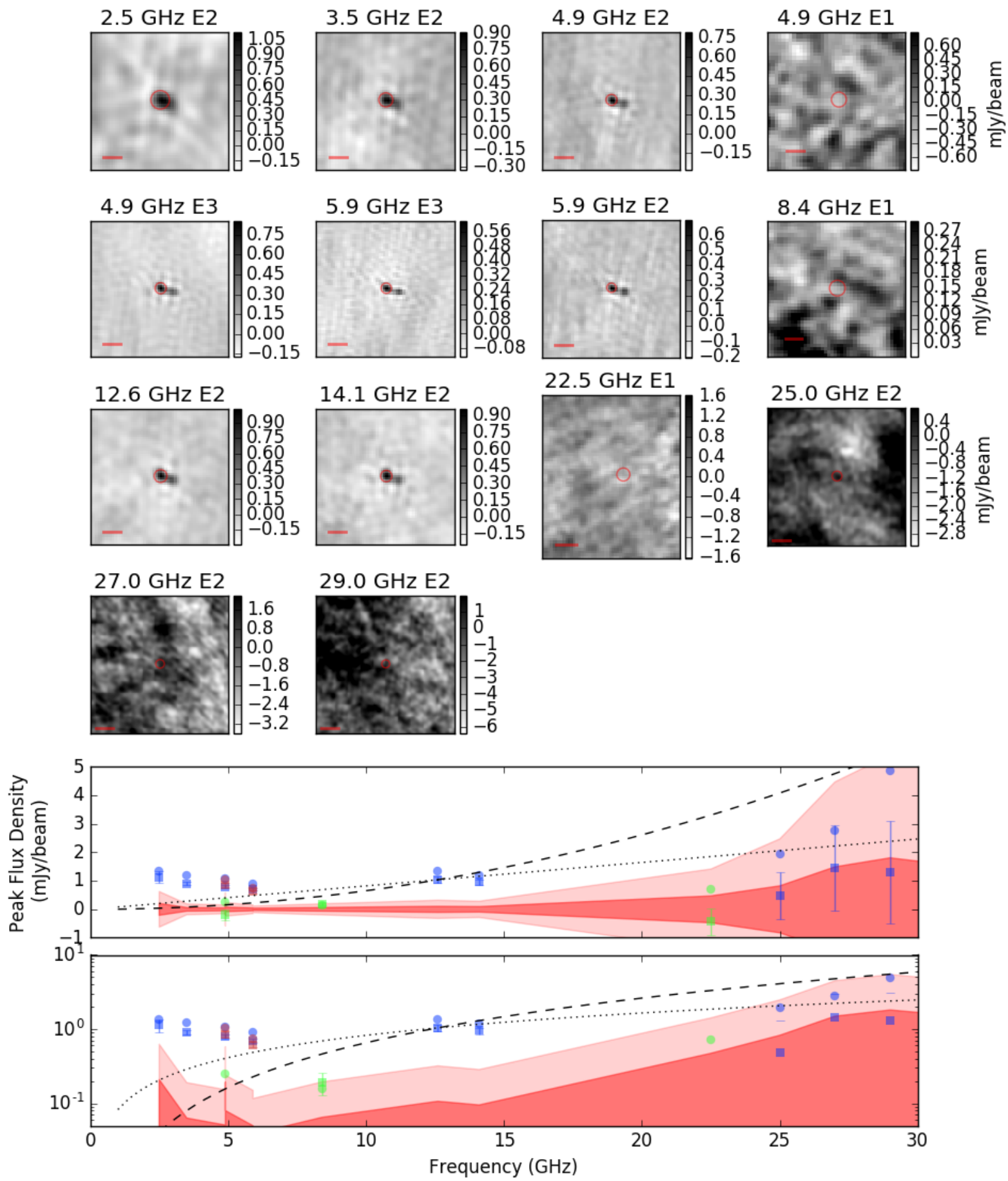

Fig. B.1. Cutouts of the source W51 d4e and its SED. The red circle shows the source aperture used to measure the peak and total flux density. The red line shows a 1" scalebar. The Epochs are indicated as E1, E2, and E3. In the SED plot, the dark and light red show the $1 \sigma$ and $3 \sigma$ noise levels. The SED plot is shown twice, first linear-linear, and second linear-log. Green points are from Epoch 1, blue points are Epoch 2, and red points are Epoch 3. Squares show the peak flux density, circles show the 'background-subtracted' flux density - i.e., the peak minus the minimum value in the map. The dotted curve shows a linear SED $\left(S_{v} \propto v\right)$ and the dashed shows a power-law curve with $\alpha=2$, i.e., $S_{v} \propto v^{2}$. Both curves are normalized to go through the $12.1 \mathrm{GHz}$ Epoch 2 data point. In this case, all of the Epoch 1 (green) points are within the noise, while all of the Epoch 2 and 3 (blue and red) points are detected at $>5 \sigma$ below $15 \mathrm{GHz}$. 


\section{Appendix C: Observation metadata}

The observation metadata is summarized in Table C.1.

Table C.1. Observation metadata.

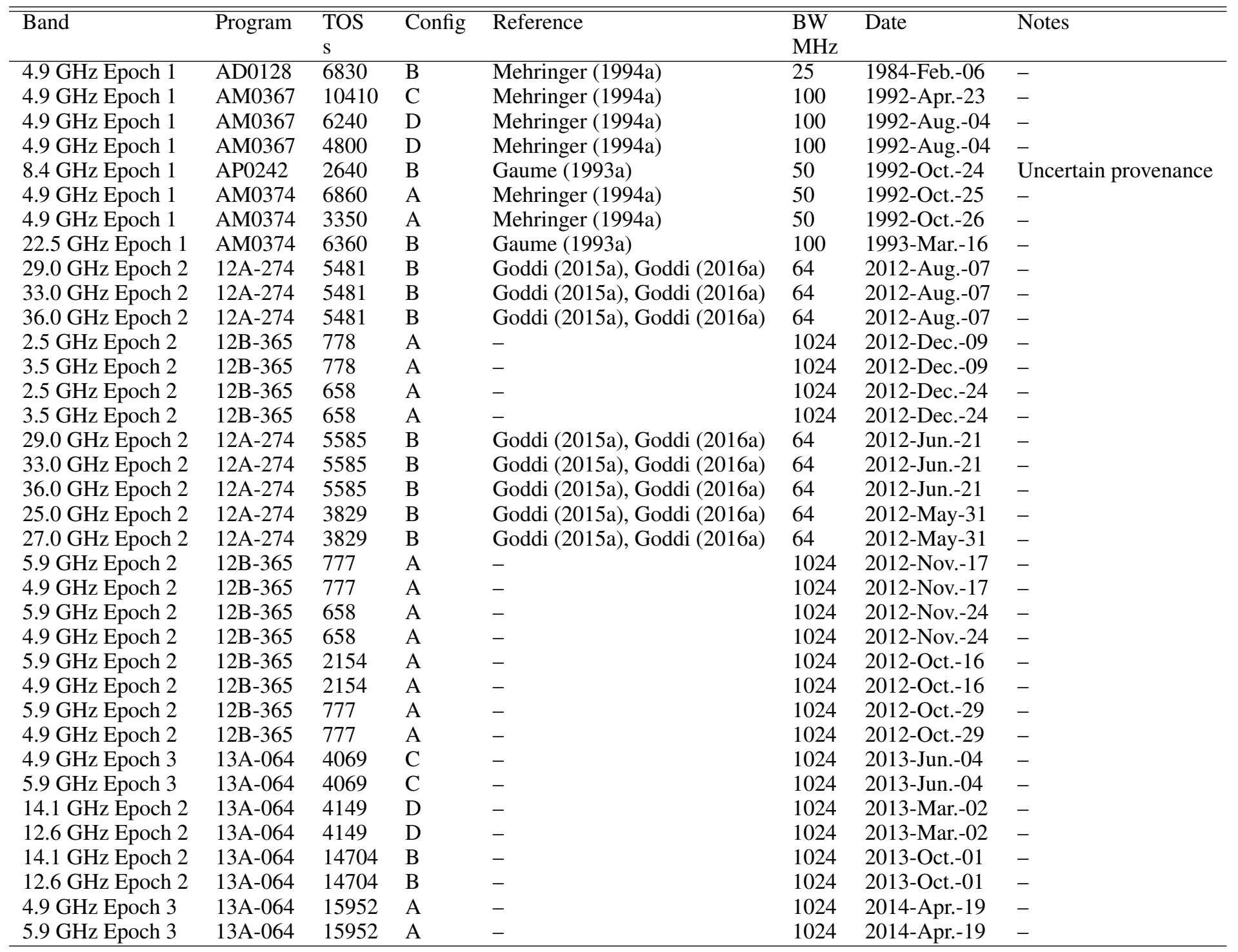




\section{Appendix D: Velocity field in W51e2}

Many works have examined the velocity field of the gas in W51e2 (Zhang \& Ho 1997; Keto \& Klaassen 2008; Shi et al. 2010a,b; Goddi et al. 2016). For comparison, we show the velocity field of a $6^{\prime \prime} \times 6^{\prime \prime}$ region centered on W51e2 in Fig. D.1.
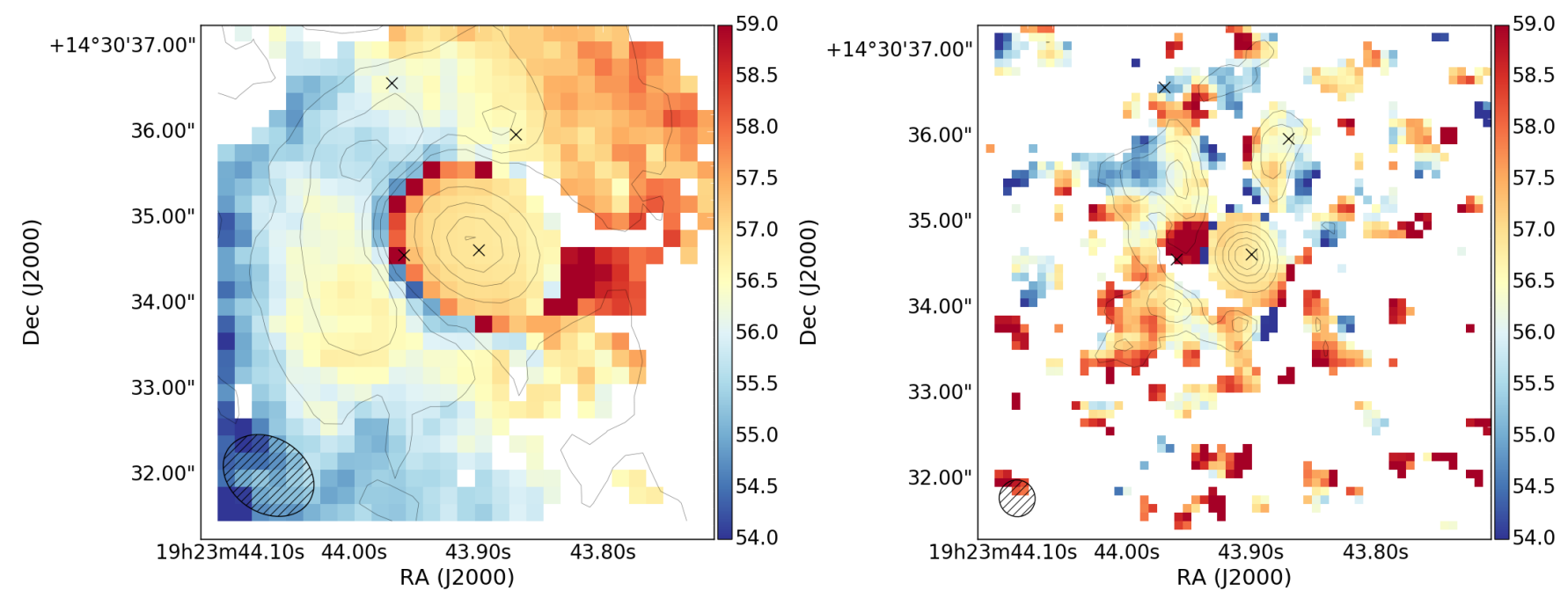

Fig. D.1. Velocity (moment 1 ) maps of $\mathrm{o}-\mathrm{H}_{2} \mathrm{CO} 2_{1,1}-2_{1,2}$ in the W51e2 region using a) the naturally weighted map and $\mathbf{b}$ ) the Briggs $w=0.5$ weighted map. The X's mark Shi et al. (2010b) cores. The contours show the moment 0 maps integrated from 50 to $63 \mathrm{~km} \mathrm{~s}^{-1}$ at $[-0.4,-0.3,-0.2$, $-0.1,0.010,0.020,0.030,0.040] \mathrm{Jy} \mathrm{km} \mathrm{s}^{-1}$ (natural) and $[-0.28,-0.21,-0.14,-0.07,0.007,0.0105,0.014] \mathrm{Jy} \mathrm{km} \mathrm{s}^{-1}$ (Briggs). 